\title{
Insights Into the Pathogenesis of Sweet's Syndrome
}

\author{
Michael S. Heath* and Alex G. Ortega-Loayza \\ Oregon Health and Science University, Department of Dermatology, Portland, OR, United States
}

Sweet's syndrome, also known as Acute Febrile Neutrophilic Dermatosis, is a rare inflammatory condition. It is considered to be the prototype disease of neutrophilic dermatoses, and presents with acute onset dermal neutrophilic lesions, leukocytosis, and pyrexia. Several variants have been described both clinically and histopathologically. Classifications include classic Sweet's syndrome, malignancy associated, and drug induced. The cellular and molecular mechanisms involved in Sweet's syndrome have been difficult to elucidate due to the large variety of conditions leading to a common clinical presentation. The exact pathogenesis of Sweet's syndrome is unclear; however, new discoveries have shed light on the role of inflammatory signaling, disease induction,

OPEN ACCESS

Edited by:

Angelo Valerio Marzano,

University of Milan, Italy

Reviewed by:

Luuk Hilbrands,

Radboud University Nijmegen, Netherlands

Stephanie T. Le,

University of California, Davis,

United States

${ }^{*}$ Correspondence:

Michael S. Heath

heatmi@ohsu.edu

Specialty section:

This article was submitted to

Molecular Innate Immunity,

a section of the journal

Frontiers in Immunology

Received: 07 November 2018

Accepted: 15 February 2019

Published: 12 March 2019

Citation:

Heath MS and Ortega-Loayza AG (2019) Insights Into the Pathogenesis of Sweet's Syndrome.

Front. Immunol. 10:414.

doi: 10.3389/fimmu.2019.00414 and relationship with malignancy. These findings include an improved understanding of inflammasome activation, malignant transformation into dermal infiltrating neutrophils, and genetic contributions. Continued investigations into effective treatments and targeted therapy will benefit patients and improve our molecular understanding of inflammatory diseases, including Sweet's syndrome.

Keywords: acute febrile neutrophilic dermatosis, neutrophilic dermatoses, malignancy, drug induced, autoinflammation, clonality, hematology

\section{INTRODUCTION}

Sweet's syndrome (SS) was originally described as "acute febrile neutrophilic dermatosis" by Sweet, (1). His original report was based on the clinical-pathologic presentation of 8 women who presented with acute onset fever, leukocytosis and erythematous, tender plaques with dense neutrophilic infiltration in the dermis. These patients had no evidence of infection and had rapid response to systemic corticosteroids. As additional reports of this newly described pathologic entity surfaced, the syndrome was renamed to recognize Dr. Sweet (2). Subsequent to these initial accounts, thousands of cases have been described in literature. This led to a better understanding and recognition of a multitude of clinical variants and SS classifications. Unfortunately, due to the rarity of SS, epidemiologic information including incidence is unknown.

The traditional description of tender erythematous plaques and nodules remains the prototypical presentation. However, clinical variants including localized neutrophilic dermatosis of the dorsal hands, bullous, subcutaneous, cellulitic, and necrotizing lesions have been reported (3-7). Extracutaneous manifestations have also been reported including involvement with the central nervous system, internal organs and musculoskeletal system (8-10). Histopathologic variants include histiocytoid SS and SS with vasculitis which has been hypothesized to be a secondary reaction $(11,12)$. 
SS is one pathologic entity within the broader neutrophilic dermatoses classification. Neutrophilic dermatoses include SS, pyoderma gangrenosum, neutrophilic eccrine hidradenitis, and Behçet's disease among others. Each disease has some overlapping pathophysiology with an autoinflammatory component made up of predominately neutrophilic infiltrate. Each entity is distinguished by disease chronicity, tissue involvement, and clinical appearance. Understanding the pathogenesis of SS is important from a diagnostic and therapeutic perspective. In a time of revolution in immunology and targeted therapy the pathways discovered in SS can have broader implications in additional autoinflammatory diseases as well as malignancy.

\section{DISEASE CLASSIFICATIONS AND ASSOCIATIONS}

SS has been associated with a multitude of diseases, malignancies and medications at varying frequencies (Table 1). Given the unpredictable nature of the disease, it has been difficult to reach conclusions regarding true associations and causations. The temporal relationships and frequency of concurrent processes has led to the recognition of several pathologic relationships. Some authors agree that there are three distinct variants which are important to distinguish, given differential work up and management recommendations. These three subtypes are Classic SS, Malignancy Associated SS, and Drug Induced SS and will be discussed individually and are summarized in Table $\mathbf{1 .}$

\section{Classic Sweet's Syndrome (Idiopathic Sweet's Syndrome)}

Classic SS is responsible for most SS cases and has a predilection for women. Initial presentation most frequently occurs between age 30 and 60 years (517), but has been reported in multiple pediatric patients including neonates in the first 10 days of life (518). Although considered idiopathic, it has been reported in association with infections, pregnancy, and inflammatory and autoimmune disorders among others (Table 1) (13, 30, 330, 435).

Diagnostic criteria for classic SS was proposed by Su and Liu and updated by von den Driesch $(254,519)$. Diagnosis is based on fulfilling both major criteria and two of the four minor criteria which are presented in Table 2.

\section{Drug Induced Sweet's Syndrome}

The most commonly reported drug associations are Granulocytecolony stimulating factor (G-CSF), Azathioprine, and All-trans retinoic acid (ATRA). Most other etiologies are infrequent (Table 1). Diagnostic criteria for drug induced SS was suggested by Walker and Cohen (250). It requires all five criteria summarized in Table 3 be met to establish the diagnosis.

\section{Malignancy-Associated Sweet's Syndrome}

It has been suggested that the first reported case of malignancy associated SS was published by Costello 9 years prior to Sweet's disease defining paper (520). Malignancy, both solid tumor and hematologic, have been reported in a large proportion of SS cases (Table 1) (521). Specific SS characteristics may represent an increased risk of malignancy, including subcutaneous and histiocytoid histopathologic variants (522, 523). Diagnostic criteria for malignancy associated SS is the same as classic SS, except for the substitution of "an underlying malignancy" as a minor criterion rather than "an inflammatory disease, pregnancy, vaccination or infection” $(254,519)$.

\section{PATHOGENESIS}

\section{Neutrophil Proliferation and Maturation}

Just as the associated condition and etiology of SS varies considerably, the pathogenesis is multifactorial and likely nonuniform between subtypes of the disease. The inciting activator of SS, especially classic SS, has not been determined, although cases of hematologic malignancy and initiation of granulocyte colony stimulating factors (G-CSF), all-trans retinoic acid (ATRA), and fms-like tyrosine kinase-3 (FLT3) inhibitors offer a glimpse into one mechanism. G-CSF acts within the bone marrow, serum and tissue, causing neutrophil differentiation, maturation and activation. As a response to pathogens, G-CSF is a part of the innate immune system signaling which is maladaptively elevated in inflammatory states (524). In cases of classic SS, patients with an underlying infection or autoimmunity, the pathologic increase in colony stimulating factors may be the causative agent (525, 526). Endogenously elevated G-CSF levels have been reported in multiple cases of SS, with elevations in serum concentrations correlating with clinical disease severity $(127,524)$. In vitro, SS neutrophils have high rates of apoptosis when isolated. Conversely, when cultured with serum from SS patients, the apoptosis rate is significantly decreased and neutrophil survival is significantly greater (524). This serum enhanced survival suggests elevated G-CSF among other circulating factors contribute to the disease. Both solid tumor and hematologic malignancies can produce colony stimulating factors. In malignancy-associated SS, this paraneoplastic phenomenon might represent an inciting factor in disease progression (127, 527-529). The frequency of drug-induced SS from the exogenous use of G-CSF further reinforces the causative role of G-CSF in SS (517, 530-533). After initiation of G-CSF therapy in SS associated with hematologic malignancies, it is theorized that G-CSF induces differentiation and maturation of leukemic cells which then home to the skin $(55,534)$. Similarly, ATRA induces the differentiation of promyelocytes in acute promyelocytic leukemia (APL). ATRA has been associated with developing SS in APL and the mature dermal neutrophils may be progeny from differentiated malignant cells. This is evidenced by sequential SS lesional biopsies showing gradual maturation of neutrophils in the dermis mirroring neutrophil maturation in the peripheral blood (181).

\section{Malignant Transformation}

Investigations have shown neutrophilic clonality within SS lesions suggestive of either hematologic malignancy transformation into mature dermal neutrophils or localized non-malignant neutrophil stemming from a common dysfunctional progenitor $(535,536)$. Analysis with fluorescent in situ hybridization have shown the SS lesional neutrophils exhibit the same genetic abnormalities as the underlying 


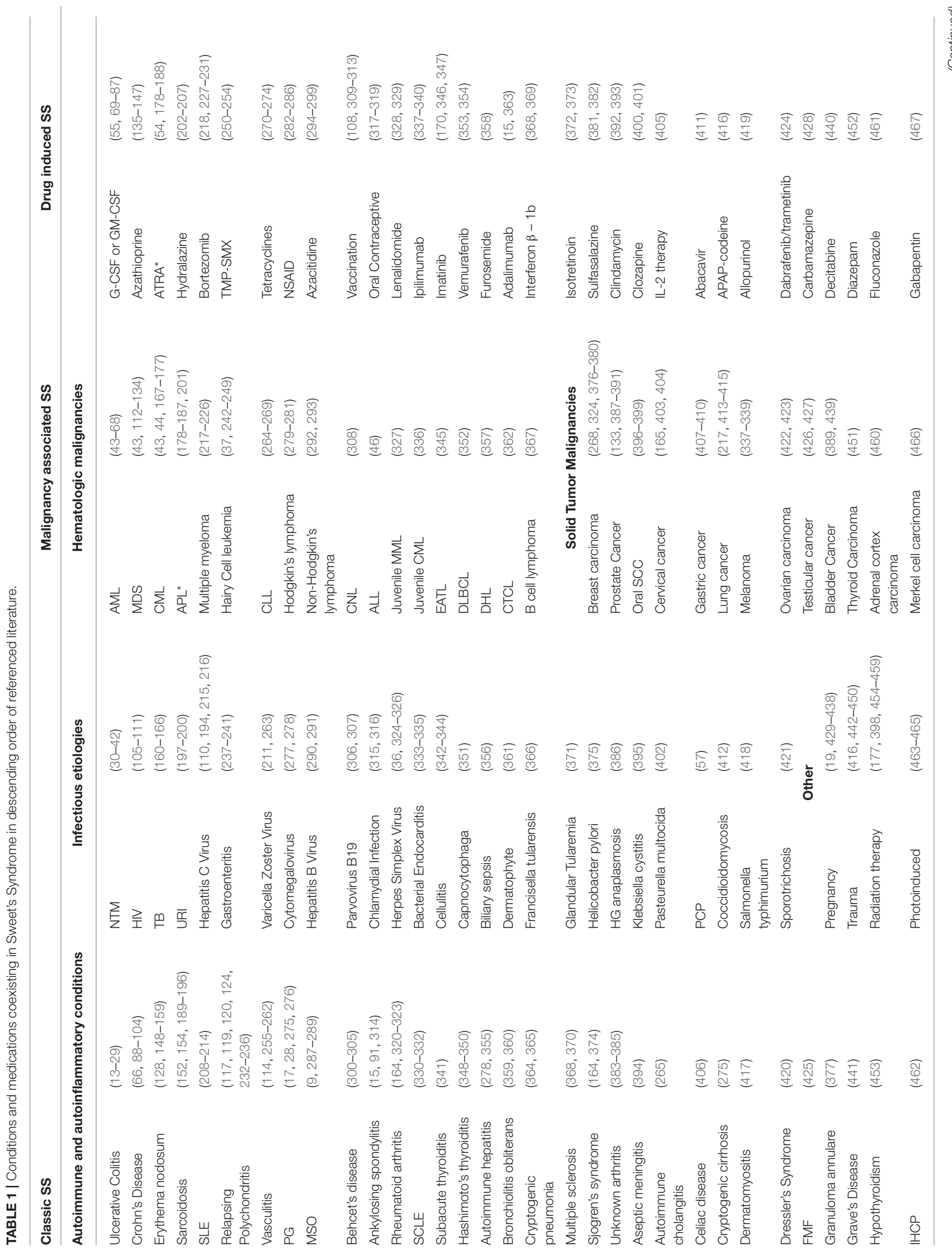




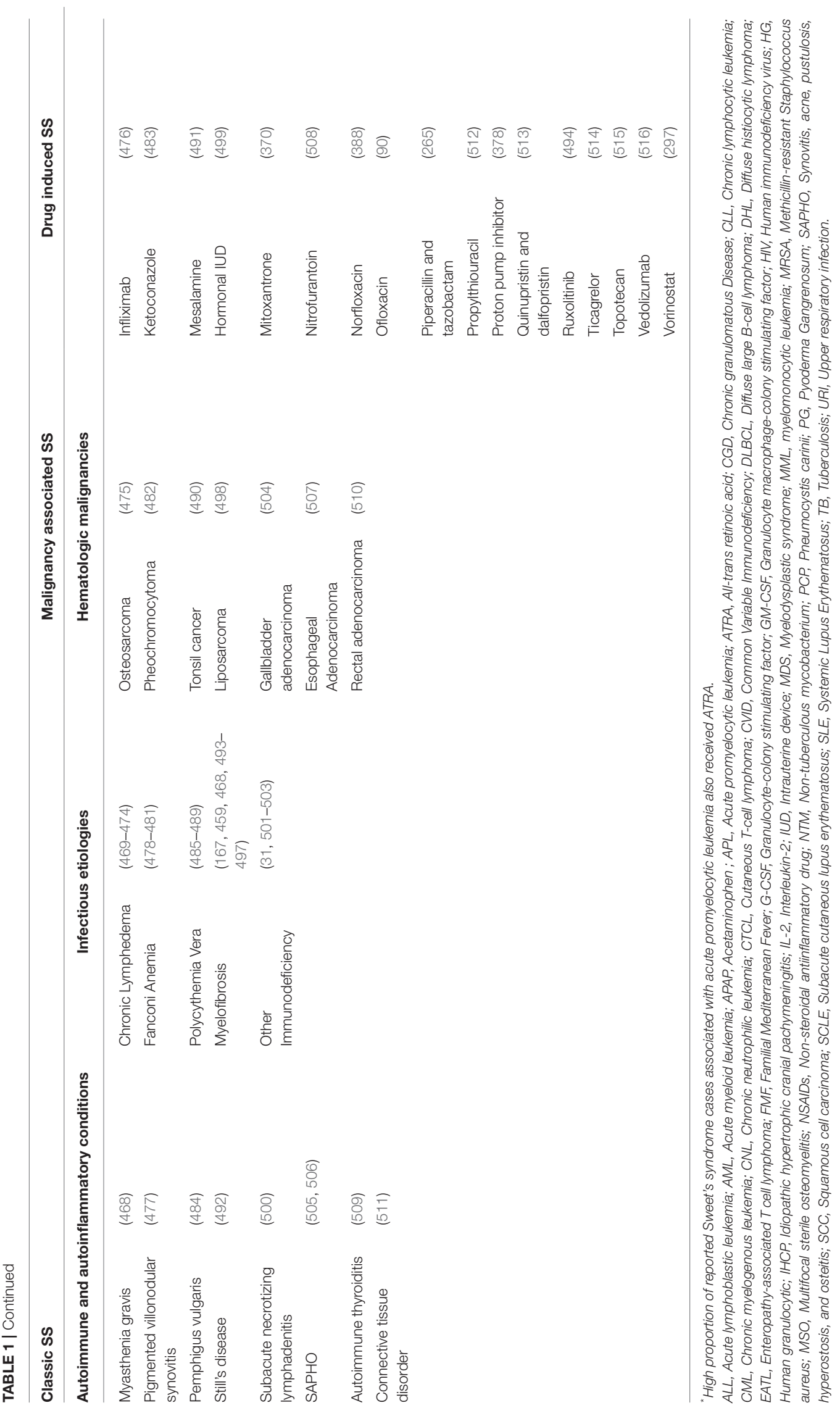


TABLE 2 | Diagnostic Criteria for Classic Sweet's Syndrome.

MAJOR CRITERIA
1. Abrupt onset of painful erythematous plaques or nodules
2. Histopathologic evidence of a dense neutrophilic infiltrate without
evidence of leukocytoclastic vasculitis
MINOR CRITERIA
1. Fever $>38^{\circ} \mathrm{C}$
2. Associated with inflammatory disease or pregnancy or preceded by
upper respiratory infection, gastrointestinal infection, or vaccination
3. Excellent response to treatment with systemic glucocorticoids or
potassium iodide
4. Abnormal laboratory values at presentation (three of four of the following):
a. Erythrocyte sedimentation rate $>20 \mathrm{~mm} / \mathrm{h}$
b. Positive C-reactive protein
c. $>8,000$ leukocytes per microliter
d. $>70 \%$ neutrophils

malignant myeloblasts in serum and bone marrow, suggesting a clonal transformation into dysplastic neutrophils in the dermis $(49,55,534,537,538)$. Recently, examination of the bone marrow and SS lesional tissue in a patient with concurrent acute myeloid leukemia (AML) with single nucleotide polymorphism array and next generation sequencing revealed FLT-3 gene mutations in infiltrating mature neutrophils and neoplastic progenitor cells (539). In one case series, FLT-3 mutations have been detected in 39\% of patients with AML and SS and FLT-3 inhibitors are a known SS inducer $(49,540,541)$. This gene encodes a receptor tyrosine kinase normally present on hematopoietic stem cells within the bone marrow and regulates myeloid progenitor cell proliferation, survival, and differentiation (542). In AML the FLT-3 mutations result in persistent activation. The identification of this mutation in dermal neutrophils and leukemic cells suggests a common progenitor origin.

\section{Induction and Stimulus}

Given the variety of underlying conditions including medications, infections, and malignancy associated with a similar clinicopathologic presentation in SS, one unifying hypothesis is that SS is a hypersensitivity reaction. Immune reaction to drugs, bacterial, viral, or tumor antigens may initiate a cytokine cascade resulting in SS (3). The efficacy of systemic corticosteroids and resolution of SS with treatment of underlying disease with antibiotics or chemotherapy supports this hypothesis, but there is a lack of evidence showing immune-complexes, immunoglobulins or changes in complement consistent with a hypersensitivity reaction $(11,519,543)$.

Photoinduction and Koebner phenomenon have also been suggested as possible inciting etiologies in SS and may explain the distribution and localization to the skin (544). Photoinduction of SS has been documented and confirmed in select patients with experimental phototesting re-challenge (464, 545-549). While not fully elucidated, a proposed mechanism is founded on the immunomodulating effects of light. The most notable concept involves the pro-inflammatory potential of ultraviolet B
TABLE 3 | Diagnostic Criteria for Drug Induced Sweet's Syndrome.

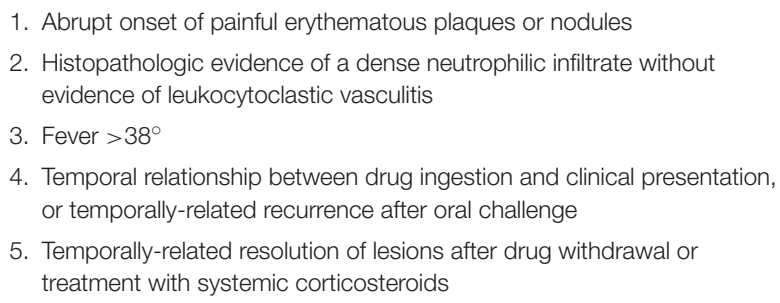

in activating neutrophils and inducing the production of TNF- $\alpha$ and interleukin- $8(548,550,551)$. The formation of SS lesions in response to localized trauma has been demonstrated by lesions developing at sites of radiation therapy, surgery, burns, tattoos, and lymphedema (442-445, 454-457, 472, 474).

\section{Cutaneous Localization}

Localization of neutrophils to the dermis in SS is complex and theorized mechanisms are dependent on underlying etiology. Normal neutrophils require TNF- $\alpha$ activated endothelium which leads to neutrophil rolling and attachment via interdependent interactions with selectins, intercellular cell adhesion molecules (ICAM), and integrins (552). These surface linking molecules in concert with inflammatory molecules, including TNF- $\alpha$ and IL-1 $\beta$, result in normal neutrophil extravasation into tissue. In hematologic malignancy, myeloid blast cells have increased expression of surface adhesion receptors and can induce non-activated endothelial cell adhesion to express receptors leading to accumulation of leukemic cells (553). These cells further promote recruitment, accumulation and tissue invasion by secreting inflammatory cytokines including TNF- $\alpha$ and IL-1 $\beta$ (553). Leukemia cutis, a paraneoplastic tissue invasion of leukemic cells, is well-recognized and has been coexistent in patients with SS and within SS lesions (554556). Potential mechanisms include dysfunctional malignant cells activating adhesions and creating an inflammatory environment suitable for innocent bystander neutrophils to extravasate, creating SS lesions. Alternatively, cancer therapy, or paraneoplastic stimulatory factors may result in the maturation of leukemia cutis cells into the mature neutrophils within SS lesions. In non-malignant SS associated with other inflammatory conditions, a similar pathologic inflammatory environment could be responsible for localization and infiltration of neutrophils.

\section{Dysfunctional Immune Mediators}

The role of a dysfunctional innate immune response in SS is well-established, but evidence is emerging that the adaptive immune system has a significant role. In classic SS, lymphocytes, specifically Type 1 helper T cells (Th1), have been theorized to be responsible for neutrophil activation and localization. This is evidenced by elevated serum levels of Th1 cytokines including IL- $1 \alpha$, IL-1 $\beta$, IL-2, and IFN- $\gamma$ (557). Further investigation utilizing immunohistochemical stains has shown a significant presence of these Th1 cytokines and a relative reduction of 
Type 2 helper $\mathrm{T}$ cell (Th2) markers in SS dermal lesions. This suggests hyperexpression of Th1 cells and a comparative suppression of Th2 cells $(137,558,559)$. Th1 cells secrete TNF- $\alpha$ and INF- $\gamma$, which are potent neutrophil recruiters and activators. Proinflammatory T helper 17 (Th17) cells and related cytokines have also been identified as a pathologic agent in SS (559-562). The role of Th17 cells is most well studied in one of the most prevalent autoinflammatory diseases: psoriasis (563). Th17 produces multiple inflammatory molecules, including interleukin 17 (IL-17). IL-17 works synergistically with TNF $\alpha$, IL- $1 \beta$, and IFN- $\gamma$ to create an inflammatory response and recruits and localizes neutrophils by inducing adhesion molecules, and chemoattractants such as IL-8 (564). Interactions with TNF $\alpha$ and IL-17 induces basement membrane remodeling via pericytes and neutrophils (565). In this SS driven remodeling process, matrix metalloproteinases (MMPs) are significantly upregulated. Upon inhibition of MMP-3, there is a reduction of neutrophil chemotaxis and extracellular matrix degradation (565). The production of G-CSF and GM-CSF are enhanced by IL-17, which leads to activation and proliferation of neutrophils $(566,567)$. Additional pro-inflammatory markers elevated in SS include: CD40/CD40 ligand, CD56, G-CSF, myeloperoxidase, IL-5, IL-8 IL-12, IL-13, L-selectin, MMP2, MMP-9, Sialic acid-binding immunoglobulin-type lectin (Siglec) 5, Siglec 9, Transforming growing factor $\beta$ (TGF$\beta$ ), TIMP-1, TNF $\alpha$, and VEGF $(127,524,558-560,562$, 568, 569). Significant levels of CD56, a Natural killer cell marker, CD40/CD40 ligand, and IFN- $\gamma$ may indicate the role of antigen presenting cells, as well as a cross-link between the robust innate and adaptive immune response in SS (570). Further evidence of adaptive immunity involvement is suggested by SS remission following treatment with therapies targeting adaptive cell processes including corticosteroids, cyclosporine, IVIG, rituximab, and vedolizumab (121, 132, 571-576). Table 4 summarizes cytokines and inflammatory markers documented in SS. Figure 1 shows the proposed multifactorial mechanism of disease.

\section{Genetic Contributions}

There is a growing body of knowledge regarding the genetic contributions in neutrophilic dermatoses including SS. Genetic susceptibility to the SS variant, neutrophilic dermatosis of the dorsal hands, in HLA-B54 positive Japanese individuals has been reported (577). Additional evidence of genetic cosusceptibility and possible mechanisms of SS have been described in synovitis, acne, pustulosis, hyperostosis, osteitis (SAPHO) syndrome, chronic recurrent multifocal osteomyelitis (CRMO), and Majeed syndrome (289, 506, 578, 579). There have been several links between SS and Familial Mediterranean fever (FMF) $(425,580)$. FMF is an inherited disease in which mutations in the MEFV gene. The MEFV gene is the causative defect identified in FMF, and it is responsible for the expression of pyrin (581). In a non-pathologic state, pyrin, an intracellular pattern recognition receptor, forms the inflammasome complex in response to infections or changes in cellular homeostasis, leading to splicing and secretion of IL-1 $\beta$ (581, 582). Mutations to MEFV as seen in FMF and
TABLE 4 | Inflammatory and signaling molecules elevated within lesional dermis and serum.

\begin{tabular}{llll}
\hline Elevated in dermis & References & Elevated in serum & References \\
\hline Interleukin-1 $\beta$ & $(137,559)$ & Interleukin-1 $\alpha$ & $(557)$ \\
Interleukin-4 & $(558)$ & Interleukin-1 $\beta$ & $(557)$ \\
Interleukin-5 & $(558)$ & Interleukin-2 & $(557)$ \\
Interleukin-8 & $(559,560,562)$ & Interleukin-6 & $(127,568)$ \\
Interleukin-10 & $(561)$ & Interferon $\gamma$ & $(557)$ \\
Interleukin-12 & $(558)$ & G-CSF & $(127,524$, \\
& & & \\
Interleukin-13 & $(558)$ & TNF- $\alpha$ & \\
Interleukin-17 & $(559,560,569)$ \\
Interferon $\gamma$ & $(558)$ & & \\
MMP-2 & $(559,560,562)$ & & \\
MMP-9 & $(560,562)$ & \\
Myeloperoxidase & $(560,562)$ & \\
Siglec 5 & $(559)$ & \\
Siglec 9 & $(559)$ & \\
TGF- $\beta$ & $(561)$ & $(559,560,562)$ & \\
TNF- $\alpha$ & $(559)$ & \\
TIMP-1 & $(560,562)$ & \\
VEGF & & \\
\end{tabular}

G-CSF, Granulocyte-colony stimulating factor; MMP, Matrix metalloproteinase; Siglec, Sialic acid-binding immunoglobulin-type lectin; TGF- $\beta$, Transforming growth factor- $\beta$; TNF- $\alpha$, Tumor necrosis factor- $\alpha$; VEGF, Vascular endothelial growth factor.

neutrophilic dermatoses leads to a pathogenic inflammatory response. FMF and SS have coexisted in the same patients and genetic analysis has revealed heterozygous mutations of MEFV in SS $(425,580)$.

Mutations in isocitrate dehydrogenase 1 (IDH1) have been identified as a possible connection to SS pathogenesis in malignancy (583). IDH1 catalyzes reactions leading to alterations in histones and DNA, causing differential gene expression (584). In myeloproliferative diseases mutations to IDH1 leads to epigenetic chaos as a result of DNA hypermethylation, which leads to abnormal transcription of numerous genes (583). Protein tyrosine phosphatase non-receptor type 6 (PTPN6) plays an essential role in the proliferation and signaling of cells within the immune system (585). Mutations leading to the disruption of normal function of PTPN6 have been identified in hematologic malignancies and neutrophilic dermatoses in mice models (586-590). Alteration of PTPN6 has also been identified in SS patients through DNA sequencing analysis (591). The evidence to date suggests that SS is a polygenic process but dysfunctional activation of the inflammasome and IL-1 $\beta$ pathway offers a unifying mechanism.

\section{Model of Pathogenesis}

The pathogenesis of SS is complex and multifactorial, the different components discussed do not provide a unifying pathway. The most complete model is within the subset of SS patients with hematologic malignancies. The pre-existing myeloid dysfunction and disruption 


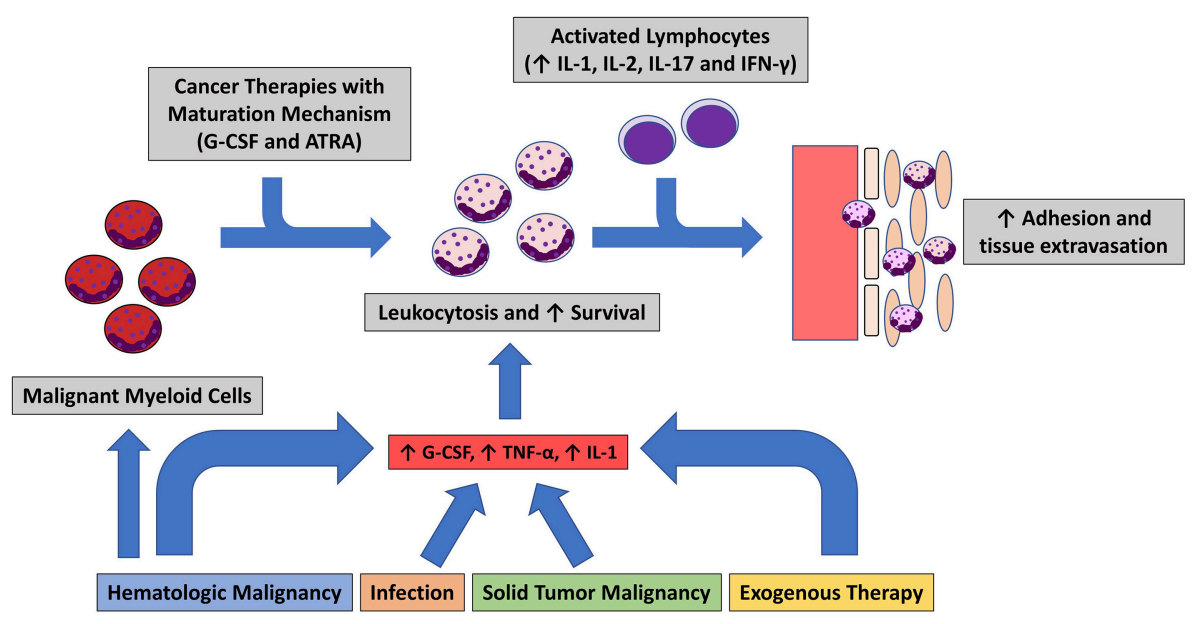

FIGURE 1 | Summary of the hypothesized of Sweet's Syndrome Pathogenesis. Inciting event leads to inflammatory state in which neutrophils mature and proliferate. Lymphocytic cytokine response leads to dermal localization.

in normal cytokine and stimulating factors provide the environment necessary for aberrant neutrophil activation and inflammation. When patients with hematologic malignancies undergoing treatment develop SS a proposed mechanism is transformation and maturation of dysfunction leukemic cells which continue to exhibit inappropriate activity. In classic SS and drug-induced SS, an inciting stimulus such as an antigen in an individual with a genetical predisposition likely creates a similar pro-inflammatory state resulting in SS. The rarity of SS and the lack of robust experimentation is a major restraint in understanding the disease pathogenesis.

\section{TREATMENT APPROACHES}

Management of SS is partially reliant on the underlying association, but given the severe presentation and possibility of non-modifiable etiology, prompt treatment is usually warranted (592). In drug induced SS, identification and removal of the offending agent is beneficial but does not negate the need for treatment. First line treatments for SS include corticosteroids and other agents such as potassium iodide or colchicine. Second line agents for SS include indomethacin, clofazimine, cyclosporin, and dapsone $(592,593)$. The effectiveness of these medications with differential mechanisms of action highlights the role of both adaptive and innate cells in the pathogenesis of SS (594-596). With advances in our understanding of the pathophysiology of neutrophilic dermatoses, especially the role of TNF- $\alpha$ and IL- $1 \beta$, the use of targeted therapy with IL- 1 and TNF- $\alpha$ inhibitors has been effective $(323,593,597-603)$. There have been reports of several novel treatments for SS, including granulocyte and monocyte adsorption apheresis, but due to the rarity of SS and the effectiveness of established treatments there have been limited investigations into these alternative treatments (604).

\section{CONCLUSIONS AND FUTURE DIRECTIONS OF RESEARCH}

Over the last half century, SS has retained its defining characteristics while medical advances and scientific discovery have led to a better understanding of disease mechanisms and associations. The clinical similarity of SS with other neutrophilic driven autoinflammatory entities is challenging in clinical grounds as the diagnostic criteria is not applicable in atypical presentations or overlapping autoinflammatory dermatoses. Relations with medications, inflammatory diseases, and malignancy have been established and expanded on. Dermal neutrophil clonality and transformation of malignant myeloid progenitors into infiltrating neutrophils provides evidence for an etiology in myeloproliferative disease and offers insight into future directions of research. Investigations into immunologic signaling pathways have improved our understanding of the interrelationships between inflammation and disease pathogenesis. The involvement of IL-17, IL-1 $\beta$, and inflammasome activation are of great interest in neutrophilic dermatoses including the utilization of targeted therapies. As this pathway is ubiquitous throughout inflammatory processes, an emphasis on better understanding its mechanism will be paramount to advances in not only SS but throughout medicine. As genetic analysis and gene profiling techniques are revolutionized and optimized, new discoveries on the role of genetic susceptibility, heritability, and more specific markers of neutrophilic dermatoses will be on the horizon

\section{AUTHOR CONTRIBUTIONS}

$\mathrm{MH}$ and $\mathrm{AO}-\mathrm{L}$ conceived the idea for this work and performed the literature review on the subject. $\mathrm{MH}$ compiled the data with AO-L oversight. $\mathrm{MH}$ and AO-L wrote the manuscript and finalized the published version. 


\section{REFERENCES}

1. Sweet R. An acute febrile neutrophtlic Dermatosts. Br J Dermatol. (1964) 76:349-56. doi: 10.1111/j.1365-2133.1964.tb14541.x

2. Howard WC, A. BG, H. CR. Recurrent neutrophilic dermatosis of the face-a variant of Sweet's Syndrome. Br J Dermatol. (1968) 80:806-10. doi: 10.1111/j.1365-2133.1968.tb11950.x

3. Voelter-Mahlknecht S, Bauer J, Metzler G, Fierlbeck G, Rassner G. Bullous variant of Sweet's syndrome. Int J Dermatol. (2005) 44:946-7. doi: 10.1111/j.1365-4632.2004.02287.x

4. Cohen PR. Subcutaneous Sweet's syndrome: a variant of acute febrile neutrophilic dermatosis that is included in the histopathologic differential diagnosis of neutrophilic panniculitis. J Am Acad Dermatol. (2005) 52:927-8. doi: 10.1016/j.jaad.2005.03.001

5. Surovy AM, Pelivani N, Hegyi I, Buettiker U, Beltraminelli H, Borradori L. Giant cellulitis-like Sweet Syndrome, a new variant of neutrophilic dermatosis. JAMA Dermatol. (2013) 149:79-83. doi: 10.1001/2013.jamadermatol.548

6. Kroshinsky D, Alloo A, Rothschild B, Cummins J, Tan J, Montecino R, et al. Necrotizing Sweet syndrome: a new variant of neutrophilic dermatosis mimicking necrotizing fasciitis. J Am Acad Dermatol. (2012) 67:945-54. doi: 10.1016/j.jaad.2012.02.024

7. Paparone PP, Paparone PA, Senyatso RY. Neutrophilic dermatosis of the dorsal hand. Wounds. (2013) 25:148-52.

8. Noda K, Okuma Y, Fukae J, Fujishima K, Goto K, Sadamasa H, et al. Sweet's syndrome associated with encephalitis. J Neurol Sci. (2001) 188:95-7. doi: 10.1016/S0022-510X(01)00541-X

9. Edwards TC, Stapleton FB, Bond MJ, Barrett FF. Sweet's syndrome with multifocal sterile osteomyelitis. Am J Dis Child. (1960) 140:817-8. doi: 10.1001/archpedi.1986.02140220099042

10. Fernandez-Bussy S, Labarca G, Cabello F, Cabello H, Folch E, Majid A. Sweet's syndrome with pulmonary involvement: case report and literature review. Respir Med Case Rep. (2012) 6:16-9. doi: 10.1016/j.rmcr.2012.08.004

11. Malone JC, Slone SP, Wills-Frank LA, Fearneyhough PK, Lear SC, Goldsmith LJ, et al. Vascular inflammation (vasculitis) in sweet syndrome: a clinicopathologic study of 28 biopsy specimens from 21 patients. Arch Dermatol. (2002) 138:345-9. doi: 10.1001/archderm.138.3.345

12. Magro CM, Momtahen S, Nguyen GH, Wang X. Histiocytoid Sweet's Syndrome: a localized cutaneous proliferation of macrophages frequently associated with chronic myeloproliferative disease. Eur J Dermatol. (2015) 25:335-41. doi: 10.1684/ejd.2015.2586

13. Stevenson R, Hannay J. Sweet's syndrome: a rare extraintestinal manifestation of ulcerative colitis. BMJ Case Rep. (2016) 11:bcr2016215524. doi: 10.1136/bcr-2016-215524

14. Lopes Cacola R, Soares M, Cardoso C, Furtado A. Sweet's syndrome complicating ulcerative colitis: a rare association. BMJ Case Rep. (2016) 20:20. doi: 10.1136/bcr-2015-212990

15. Bruscino N, Grandi V, Gunnella S, Maio V. Sweet's syndrome in a patient affected by ankylosing spondylitis and ulcerative colitis under treatment with adalimumab. J Eur Acad Dermatol Venereol. (2016) 30:195-6. doi: $10.1111 /$ jdv. 12710

16. Bancu LA, Ureche C, Craciun NM, Marian D. A case of Sweet's syndrome associated with uveitis in a young male with ulcerative colitis. Roman J Morpholo Embryol. (2016) 57:1145-7.

17. Ajili F, Souissi A, Bougrine F, Boussetta N, Abdelhafidh NB, Sayhi $\mathrm{S}$, et al. Coexistence of pyoderma gangrenosum and sweet's syndrome in a patient with ulcerative colitis. Pan Afr Med J. (2015) 21:151. doi: 10.11604/pamj.2015.21.151.6364

18. Shin OR, Lee YH, Bak SH, Cho YS. Gastroenterology: Sweet's syndrome in a patient with acutely exacerbated ulcerative colitis. J Gastroenterol Hepatol. (2015) 30:965. doi: 10.1111/jgh.12911

19. Best J, Dechene A, Esser S, Gerken G, Canbay A. Pregnancy-associated Sweet's syndrome in an acute episode of ulcerative colitis. Zeitschrift fur Gastroenterol. (2009) 47:753-7. doi: 10.1055/s-0028-1109423

20. Kang W, Hao C, Nie Q. Clinical challenges and images in GI. Sweet syndrome in association with ulcerative colitis. [Erratum appears in Gastroenterology. 2009 Jul;137:393]. Gastroenterology. (2009) 136:15071846. doi: $10.1053 /$ j.gastro. 2008
21. Ali M, Duerksen DR. Ulcerative colitis and Sweet's syndrome: a case report and review of the literature. Can J Gastroenterol. (2008) 22:296-8. doi: 10.1155/2008/960585

22. Castro-Fernandez M, Sanchez-Munoz D, Ruiz-Granados E, Merchante N, Corzo J. Coexistence of pyoderma gangrenosum and Sweet's syndrome in a patient with ulcerative colitis. Am J Gastroenterol. (2007) 102:2865-6. doi: 10.1111/j.1572-0241.2007.01528_8.x

23. Malheiros AP, Teixeira MG, Takahashi MD, de Almeida MG, Kiss DR, Cecconello I. Sweet syndrome associated with ulcerative colitis. Inflamm Bowel Dis. (2007) 13:1583-4. doi: 10.1002/ibd.20227

24. Natour M, Chowers Y, Solomon M, Khaikin M, Barshack I, Ayalon A, et al. Sweet's syndrome in association with ulcerative colitis and dyserythropoietic anemia. Digestion. (2007) 75:142-3. doi: 10.1159/000106455

25. Diaz-Peromingo JA, Garcia-Suarez F, Sanchez-Leira J, Saborido-Frojan J. Sweet's syndrome in a patient with acute ulcerative colitis: presentation of a case and review of the literature. Yale J Biol Med. (2001) 74:165-8.

26. Paoluzi OA, Crispino P, Amantea A, Pica R, Iacopini F, Consolazio A, et al. Diffuse febrile dermatosis in a patient with active ulcerative colitis under treatment with steroids and azathioprine: a case of Sweet's syndrome. Case report and review of literature. Dig Liver Dis. (2004) 36:361-6. doi: 10.1016/j.dld.2003.10.020

27. Wells MM, Stecho W, Wehrli B, Khanna N. Sweet syndrome secondary to inflammatory bowel disease. Can J Gastroenterol. (2013) 27:124-5. doi: $10.1155 / 2013 / 848316$

28. Benton EC, Rutherford D, Hunter JA. Sweet's syndrome and pyoderma gangrenosum associated with ulcerative colitis. Acta Dermato-venereol. (1985) 65:77-80.

29. Terai T, Sugimoto M, Osawa S, Sugimoto K, Furuta T, Kanaoka S, et al. Successful treatment of ulcerative colitis complicated by Sweet's syndrome by corticosteroid therapy and leukocytapheresis. Clin J Gastroenterol. (2011) 4:151-6. doi: 10.1007/s12328-011-0215-z

30. Chaowattanapanit S, Choonhakarn C, Chetchotisakd P, Sawanyawisuth K, Julanon N. Clinical features and outcomes of Sweet's syndrome associated with non-tuberculous mycobacterial infection and other associated diseases. J Dermatol. (2016) 43:532-6. doi: 10.1111/1346-8138.13167

31. Wang HH, Huang YC, Chen WY, Chiang YY. Subcutaneous Sweet syndrome associated with disseminated nontuberculous mycobacterial infection leading to the diagnosis of adult-onset immunodeficiency. J Am Acad Dermatol. (2014) 71:e20-2. doi: 10.1016/j.jaad.2014.01.855

32. Choonhakarn C, Chetchotisakd P, Jirarattanapochai K, Mootsikapun P. Sweet's syndrome associated with non-tuberculous mycobacterial infection: a report of five cases. Br J Dermatol. (1998) 139:107-10. doi: 10.1046/j.1365-2133.1998.02324.x

33. Tuchinda C, Puavilai S, Sathapatayavongs B, Sungkanuparph S, Vibhagool A, Jirasutus S, et al. Sweet's syndrome: a reaction to non-tuberculous mycobacterial infections. I Med Assoc Thailand. (2004) 87:567-72.

34. Chen $\mathrm{HH}$, Hsiao $\mathrm{CH}$, Chiu HC. Successive development of cutaneous polyarteritis nodosa, leucocytoclastic vasculitis and Sweet's syndrome in a patient with cervical lymphadenitis caused by Mycobacterium fortuitum. $\mathrm{Br}$ J Dermatol. (2004) 151:1096-100. doi: 10.1111/j.1365-2133.2004.06201.x

35. Teraki Y, Ono S, Izaki S. Sweet's syndrome associated with Mycobacterium avium infection. Clin Exp Dermatol. (2008) 33:599-601. doi: $10.1111 / \mathrm{j} .1365-2230.2008 .02732 . \mathrm{x}$

36. Theng TS, Chan YC, Leow YH, Tan SH. Sweet's syndrome associated with Mycobacterium chelonae and herpes simplex virus infections: a case report. Ann Acad Med. (2003) 32:411-4.

37. Kramers C, Raemaekers JM, van Baar HM, de Pauw BE, Horrevorts AM. Sweet's syndrome as the presenting symptom of hairy cell leukemia with concomitant infection by Mycobacterium kansasii. Ann Hematol. (1992) 65:55-8. doi: 10.1007/BF01715129

38. Sprague J, Leibowitz M, Chiu MW. Cutaneous infection with Mycobacterium kansasii in a patient with myelodysplastic syndrome and Sweet syndrome. Cutis. (2015) 96:E10-2.

39. Juan CK, Shen JL, Yang CS, Liu KL, Yen CY. Sweet's syndrome associated with Mycobacterium kansasii infection in an immunocompetent woman. J der Deutschen Dermatol Gesellschaft. (2015) 13:921-3. doi: $10.1111 /$ ddg. 12635 
40. Hsiao GH, Chiu HC. Atypical mycobacterial cervical lymphadenitis associated with Sweet's syndrome. Acta dermato-venereol. (1995) 75:237-9.

41. Chetchotisakd P, Kiertiburanakul S, Mootsikapun P, Assanasen S, Chaiwarith $\mathrm{R}$, Anunnatsiri S. Disseminated nontuberculous mycobacterial infection in patients who are not infected with HIV in Thailand. Clin Infect Dis. (2007) 45:421-7. doi: $10.1086 / 520030$

42. Hibiya K, Miyagi K, Tamayose M, Nabeya D, Kinjo T, Takeshima S, et al. Do infections with disseminated Mycobacterium avium complex precede sweet's syndrome? A case report and literature review. Int J Mycobacteriol. (2017) 6:336-43. doi: 10.4103/ijmy.ijmy_172_17

43. Cho KH, Han KH, Kim SW, Youn SW, Youn JI, Kim BK. Neutrophilic dermatoses associated with myeloid malignancy. Clin Exp Dermatol. (1997) 22:269-73. doi: 10.1111/j.1365-2230.1997.tb01093.x

44. Paydas S, Sahin B, Zorludemir S. Sweet's syndrome accompanying leukaemia: seven cases and review of the literature. Leukemia Res. (2000) 24:83-6. doi: 10.1016/S0145-2126(99)00140-X

45. Colovic M, Jankovic G, Suvajdzic N, Nikolic M. Structural chromosomal abnormality of $1 \mathrm{q}$ in acute leukemia with Sweet syndrome. Cancer Genet Cytogenet. (2002) 139:84-5. doi: 10.1016/S0165-4608(02)00608-8

46. Heer-Sonderhoff AH, Arning M, Wehmeier A, Sudhoff T, Zumdick M. Neutrophilic dermal infiltrates in granulocytopenic patients with acute leukemia. Ann Hematol. (1995) 71:257-61. doi: 10.1007/BF01744376

47. Srisuttiyakorn C, Reeve J, Reddy S, Imaeda S, Lazova R. Subcutaneous histiocytoid Sweet's syndrome in a patient with myelodysplastic syndrome and acute myeloblastic leukemia. J Cutaneous Pathol. (2014) 41:475-9. doi: 10.1111/cup. 12305

48. Korekawa A, Nakajima K, Nakano H, Sawamura D. Subcutaneous histiocytoid Sweet's syndrome followed by acute myelocytic leukemia. $J$ Dermatol. (2016) 43:1370-1. doi: 10.1111/1346-8138.13401

49. Kazmi SM, Pemmaraju N, Patel KP, Cohen PR, Daver N, Tran $\mathrm{KM}$, et al. Characteristics of Sweet Syndrome in patients with acute myeloid leukemia. Clin Lymphoma Myeloma Leukemia. (2015) 15:358-63. doi: 10.1016/j.clml.2014.12.009

50. Disel U, Paydas S, Yavuz S, Tuncer I, Alpay R. Bilateral ear Sweet's syndrome in a case with relapse acute myeloblastic leukemia. Leukemia Res. (2006) 30:364. doi: 10.1016/j.leukres.2005.08.003

51. Ozcelik T, Ozkocaman V, Ali R, Ozkalemkas F, Bulbul-Baskan E, Yazici B, et al. Sweet's syndrome: dilemma in febrile neutropenic patient with acute myeloid leukemia. Leukemia Res. (2006) 30:1466-8. doi: 10.1016/j.leukres.2006.01.019

52. Maalouf T, Angioi K, Ssi-Yan-Kai I, Vernerey F, Witz B, George J. Dacryoadenitis associated with subcutaneous Sweet's syndrome in a patient with acute myeloid leukemia. Orbit. (Amsterdam, Netherlands). (2005) 24:55-7. doi: 10.1080/01676830590903562

53. Breccia M, D'Andrea M, Girmenia C, Bottoni U, Carmosino I, Biondo F, et al. Recurrent Sweet's syndrome in acute myeloid leukemia successfully treated with amphotericin B. Haematologica. (2004) 89:ELT04.

54. Al-Saad K, Khanani MF, Naqvi A, Krafchik B, Grant R, Pappo A. Sweet syndrome developing during treatment with all-trans retinoic acid in a child with acute myelogenous leukemia. J Pediatr Hematol Oncol. (2004) 26:197-9. doi: 10.1097/00043426-200403000-00011

55. Magro CM, De Moraes E, Burns F. Sweet's syndrome in the setting of CD34-positive acute myelogenous leukemia treated with granulocyte colony stimulating factor: evidence for a clonal neutrophilic dermatosis. $J$ Cutan Pathol. (2001) 28:90-6. doi: 10.1034/j.1600-0560.2001. 280205.X

56. Yoon TY, Ahn GB, Yang TH, Shin HS. Sweet's syndrome with abscess-like lesions in a patient with acute myelogenous leukemia. J Dermatol. (2000) 27:794-7. doi: 10.1111/j.1346-8138.2000.tb02284.x

57. Chowdhary V, Nityanand S, Prasad KN, Pandey R, Dabadghao S. Sweet's syndrome and Pneumocystis carinii pneumonia: two sequelae of low-dose cytosine arabinoside therapy in a patient with acute myeloid leukemia. Eur J Haematol. (2000) 65:72-3. doi: 10.1034/j.1600-0609.2000.9c177.x

58. van Mook WN, Fickers MM, van der Kley JA, Theunissen PH. A case of herpes-like Sweet's syndrome in acute myelogenous leukemia during treatment with G-CSF. Netherlands J Med. (1999) 55:235-41. doi: 10.1016/S0300-2977(99)00080-7
59. Ravaglia C, Poletti G, Gurioli C, Casoni GL, Dubini A, Re G, et al. Sweet's syndrome associated with myelogenous leukemia and pulmonary involvement. Monaldi Arch Chest Dis. (2011) 75:149-50.

60. Equitani F, Mele L, Rutella S, Belli P, Paciaroni K, Piscitelli R, et al. Atypical Sweet's syndrome in a neutropenic patient with acute myeloid leukemia, secondary to a RAEB-T, simulating thrombophlebitis. Panminerva Med. (1999) 41:261-3.

61. Schneider DT, Schuppe HC, Schwamborn D, Koerholz D, Lehmann P, Goebel U. Acute febrile neutrophilic dermatosis (Sweet syndrome) as initial presentation in a child with acute myelogenous leukemia. Med Pediatr Oncol. (1998) 31:178-81.

62. Conesa V, Morales A, Majado MJ, Gonzalez C, Candel R. Post-chemotherapy Sweet's syndrome in three patients with AML. Am J Hematol. (1998) 57:179.

63. Chiang CT, Chan HL, Kuo TT, Wang PN. Herpes zoster-like Sweet's syndrome in acute myelogenous leukemia. Int J Dermatol. (1997) 36:717-8.

64. Deguchi M, Tsunoda T, Yuda F, Tagami H. Sweet's syndrome in acute myelogenous leukemia showing dermal infiltration of leukemic cells. Dermatology. (1997) 194:182-4. doi: 10.1159/000246094

65. Genet P, Pulik M, Lionnet F, Petitdidier C, Petit A, Gaulier A. Sweet's syndrome during acute myeloid leukaemia: is there a role for hematopoietic growth factors? Am J Hematol. (1995) 50:64. doi: 10.1002/ajh.2830500115

66. Banet DE, McClave SA, Callen JP. Oral metronidazole, an effective treatment for Sweet's syndrome in a patient with associated inflammatory bowel disease. J Rheumatol. (1994) 21:1766-8.

67. Dompmartin A, Troussard X, Lorier E, Jacobs F, Reman O, Leroy $\mathrm{D}$, et al. Sweet syndrome associated with acute myelogenous leukemia. Atypical form simulating facial erysipelas. Int J Dermatol. (1991) 30:644-7. doi: 10.1111/j.1365-4362.1991.tb03491.x

68. Tercedor J, Rodenas JM, Henraz MT, Garcia-Mellado V, GutierrezSalmeron MT, Naranjo R. Facial cellulitis-like Sweet's syndrome in acute myelogenous leukemia. Int J Dermatol. (1992) 31:598-9. doi: 10.1111/j.1365-4362.1992.tb02731.x

69. Akilov OE, Desai N, Jaffe R, Gehris RP. Bullous Sweet's syndrome after granulocyte colony-stimulating factor therapy in a child with congenital neutropenia. Pediatr Dermatol. (2014) 31:e61-2. doi: 10.1111/pde.12282

70. Bidyasar S, Montoya M, Suleman K, Markowitz AB. Sweet syndrome associated with granulocyte colony-stimulating factor. [Erratum appears in J Clin Oncol. 2008 Nov 20;26:5493]. J Clin Oncol. (2008) 26:4355-6.

71. Oiso N, Watanabe K, Kawada A. Granulocyte colony-stimulating factorinduced Sweet syndrome in a healthy donor. Br J Haematol. (2006) 135:148. doi: $10.1111 / j .1365-2141.2006 .06222 . x$

72. Thompson MA, Dyson SW, Faderl S. Sweet's syndrome in chronic lymphocytic leukemia associated with neutropenic fever and granulocyte colony stimulation factor. Am J Hematol. (2006) 81:703-5. doi: 10.1002/ajh.20666

73. Park JW, Mehrotra B, Barnett BO, Baron AD, Venook AP. The Sweet syndrome during therapy with granulocyte colony-stimulating factor. Ann Internal Med. (1992) 116(12 Pt 1):996-8. doi: 10.7326/0003-4819-116-12-996

74. Paydas S, Sahin B, Seyrek E, Soylu M, Gonlusen G, Acar A, et al. Sweet's syndrome associated with G-CSF. Br J Haematol. (1993) 85:191-2. doi: 10.1111/j.1365-2141.1993.tb08668.x

75. Jain KK. Sweet's syndrome associated with granulocyte colony-stimulating factor. Cutis. (1996) 57:107-10.

76. Garty BZ, Levy I, Nitzan M, Barak Y. Sweet syndrome associated with GCSF treatment in a child with glycogen storage disease type Ib. Pediatrics. (1996) 97:401-3

77. Petit T, Frances C, Marinho E, Herson S, Chosidow O. Lymphoedema-arearestricted Sweet syndrome during G-CSF treatment. Lancet. (1996) 347:690. doi: 10.1016/S0140-6736(96)91241-6

78. Shimizu T, Yoshida I, Eguchi H, Takahashi K, Inada H, Kato H. Sweet syndrome in a child with aplastic anemia receiving recombinant granulocyte colony-stimulating factor. J Pediatr Hematol Oncol. (1996) 18:282-4. doi: 10.1097/00043426-199608000-00009

79. Richard MA, Grob JJ, Laurans R, Hesse S, Brunet P, Stoppa AM, et al. Sweet's syndrome induced by granulocyte colony-stimulating factor in a woman with congenital neutropenia. J Am Acad Dermatol. (1996) 35:629-31. doi: 10.1016/S0190-9622(96)90693-3 
80. Prevost-Blank PL, Shwayder TA. Sweet's syndrome secondary to granulocyte colony-stimulating factor. J Am Acad Dermatol. (1996) 35:995-7. doi: 10.1016/S0190-9622(96)90132-2

81. Chao SC, Lee JY, Tsao CJ. Sweet's syndrome in a severely neutropenic patient during therapy with recombinant human granulocyte colony-stimulating factor. J Formosan Med Assoc. (1997) 96:276-9.

82. Hasegawa M, Sato S, Nakada M, Nitta H, Shirasaki H, Kasahara K, et al. Sweet's syndrome associated with granulocyte colony-stimulating factor. Eur J Dermatol. (1998) 8:503-5.

83. Arbetter KR, Hubbard KW, Markovic SN, Gibson LE, Phyliky RL. Case of granulocyte colony-stimulating factor-induced Sweet's syndrome. Am J Hematol. (1999) 61:126-9.

84. Kumar G, Bernstein JM, Waibel JS, Baumann MA. Sweet's syndrome associated with sargramostim (granulocyte-macrophage colony stimulating factor) treatment. Am J Hematol. (2004) 76:283-5. doi: 10.1002/ajh.20112

85. Fukutoku M, Shimizu S, Ogawa Y, Takeshita S, Masaki Y, Arai T, et al. Sweet's syndrome during therapy with granulocyte colony-stimulating factor in a patient with aplastic anaemia. Br J Haematol. (1994) 86:645-8. doi: 10.1111/j.1365-2141.1994.tb04799.x

86. Llamas-Velasco M, Garcia-Martin P, Sanchez-Perez J, Fraga J, GarciaDiez A. Sweet's syndrome with subcutaneous involvement associated with pegfilgrastim treatment: first reported case. J Cutaneous Pathol. (2013) 40:46-9. doi: 10.1111/cup.12042

87. Machan M, Matthys B, Fraga GR. Pegfilgrastim-induced Sweet's syndrome: a case report. Int J Dermatol. (2014) 53:1275-7. doi: 10.1111/j.1365-4632.2012.05744.x

88. Lima CDS, Pinto RDB, Goes HFO, Salles SAN, Vilar EAG, Lima CDS. Sweet's syndrome associated with Crohn's disease. Anais Brasileiros de Dermatol. (2017) 92:263-5. doi: 10.1590/abd1806-4841.20175298

89. Ortega-Alonso A, Lopez-Navarro N, Gallego E, Lara C, Alcain-Martinez G. Crohn's disease and Sweet's syndrome: a debut together. Rev Espanola de Enfermedades Digest. (2015) 107:765-6. doi: 10.17235/reed.2015.3842/2015

90. Ozdemir D, Korkmaz U, Sahin I, Sencan I, Kavak A, Kucukbayrak A, et al. Ofloxacin induced Sweet's syndrome in a patient with Crohn's disease. J Infect. (2006) 52:e155-7. doi: 10.1016/j.jinf.2005.08.002

91. Petermann A, Tebbe B, Distler A, Sieper J, Braun J. Sweet's syndrome in a patient with acute Crohn's colitis and longstanding ankylosing spondylitis. Clin Exp Rheumatol. (1999) 17:607-10.

92. Beitner H, Nakatani T, Hammar H. A case report of acute febril neutrophilic dermatosis (Sweet's syndrome) and Crohn's disease. Acta Dermato-Venereol. (1991) 71:360-3.

93. Burrows NP. Sweet's syndrome in association with Crohn's disease. Clin Exp Dermatol. (1995) 20:279-80. doi: 10.1111/j.1365-2230.1995.tb01324.x

94. Actis GC, Lagget M, Ciancio A, Rocca G, Tomasini C, Puiatti P, et al. Recurrent Sweet's syndrome in reactivated Crohn's disease. J Clin Gastroenterol. (1995) 21:317-9. doi: 10.1097/00004836-199512000-00015

95. Carpels W, Mattelaer C, Geboes K, Coremans G, Tack J. Sweet's syndrome in a patient with Crohn's disease. Acta Gastroenterol Belgica. (1999) 62:372-4.

96. Vaz A, Kramer K, Kalish RA. Sweet's syndrome in association with Crohn's disease. Postgr Med J. (2000) 76:713-4. doi: 10.1136/pmj.76.901.713

97. Rappaport A, Shaked M, Landau M, Dolev E. Sweet's syndrome in association with Crohn's disease: report of a case and review of the literature. Dis Colon and Rectum. (2001) 44:1526-9. doi: 10.1007/BF02234611

98. Catalan-Serra I, Martin-Moraleda L, Navarro-Lopez L, Gil-Borras R, PontSanjuan V, Ferrando-Marco J, et al. Crohn's disease and Sweet's syndrome: an uncommon association. Rev Espanola de Enfermedades Digest. (2010) 102:331-7. doi: 10.4321/S1130-01082010000500009

99. Fernandez-Torres RM, Castro S, Moreno A, Alvarez R, Fonseca E. Subcutaneous histiocytoid sweet syndrome associated with crohn disease in an adolescent. Case Rep Dermatol Med. (2014) 2014:954254. doi: 10.1155/2014/954254

100. Hiari N, Borland C. A 47-year-old man with neuro-Sweet syndrome in association with Crohn's disease: a case report. J Med Case Rep. (2009) 3:8997. doi: 10.4076/1752-1947-3-8997

101. Anwar S, Hishamuddin A, Khairul AJ, Shamsul AJ, Razlan H, Said MS. Sweet's syndrome in Crohn's colitis. BMJ Case Rep. (2009) 2009:1-5. doi: 10.1136/bcr.01.2009.1438
102. Mustafa NM, Lavizzo M. Sweet's syndrome in a patient with Crohn's disease: a case report. J Med Case Rep. (2008) 2:221. doi: 10.1186/1752-1947-2-221

103. Becuwe C, Delaporte E, Colombel JF, Piette F, Cortot A, Bergoend H. Sweet's syndrome associated with Crohn's disease. Acta Dermato Venereol. (1989) 69:444-5.

104. Kemmett D, Gawkrodger DJ, Wilson G, Hunter JA. Sweet's syndrome in Crohn's disease. BMJ. (1988) 297:1513-4. doi: 10.1136/bmj.297.6662.1513-a

105. Hilliquin P, Marre JP, Cormier C, Renoux M, Menkes CJ, Puissant A. Sweet's syndrome and monarthritis in a human immunodeficiency virus-positive patient. Arth Rheum. (1992) 35:484-6. doi: 10.1002/art.1780350423

106. Bevilacqua S, Hermans P, Van Laethem Y, Demaubeuge J, Clumeck N. Sweet's syndrome in an HIV-infected patient. AIDS. (1999) 13:728-9. doi: 10.1097/00002030-199904160-00015

107. Brady RC, Morris J, Connelly BL, Boiko S. Sweet's syndrome as an initial manifestation of pediatric human immunodeficiency virus infection. Pediatrics. (1999) 104(5 Pt 1):1142-4. doi: 10.1542/peds.104.5.1142

108. Tan AW, Tan HH, Lim PL. Bullous Sweet's syndrome following influenza vaccination in a HIV-infected patient. Int J Dermatol.. (2006) 45:1254-5. doi: 10.1111/j.1365-4632.2006.03005.x

109. Inamadar AC, Anitha B. HIV-seropositive patient with Sweet's syndrome and nodular scleritis, showing dramatic response after adding dapsone to systemic corticosteroid therapy. Int J Dermatol. (2008) 47:836-8. doi: 10.1111/j.1365-4632.2008.03648.x

110. Deasy AM, Walker B, Layton AM, Lacey CJ. Sweet's syndrome in a patient with haemophilia, HIV and hepatitis C infection. Int J STD AIDS. (2012) 23:e11-3. doi: 10.1258/ijsa.2009.009314

111. Rajendran A, Zacharia GS, Zacharia SA, George KC. Sweet's syndrome in human immune deficiency virus-infected patient. Indian journal of sexually transmitted diseases and AIDS. (2014) 35:149-51. doi: 10.4103/0253-7184.142413

112. Li Y, Ai M, Yang WB, Li X. Vital organ involvement in Sweet's syndrome with myelodysplastic syndrome: a case report and literature review. Int J Dermatol. (2015) 54:1303-8. doi: 10.1111/ijd.12280

113. Kulasekararaj AG, Kordasti S, Basu T, Salisbury JR, Mufti GJ, du Vivier AW. Chronic relapsing remitting Sweet syndrome-a harbinger of myelodysplastic syndrome. Br J Haematol. (2015) 170:649-56. doi: 10.1111/bjh.13485

114. Pinal-Fernandez I, Ferrer Fabrega B, Ramentol Sintas M, Solans Laque R. Histiocytoid Sweet syndrome and cutaneous polyarteritis nodosa secondary to myelodysplastic syndrome. Int J Rheum Dis. (2013) 16:777-9. doi: 10.1111/1756-185X.12103

115. Ono S, Otsuka A, Kabashima K, Miyachi Y, Tachibana T. Sweet's syndrome presenting as drastically spreading generalized erythema with subcorneal pustulosis in myelodysplastic syndrome. J Dermatol. (2013) 40:1072-3. doi: 10.1111/1346-8138.12344

116. Reina D, Cerda D, Roig D, Figuls R, Villegas ML, Corominas H. Sweet syndrome associated with myelodysplastic syndrome: report of a case. Review of the literature. Reumatol Clin. (2013) 9:246-7. doi: 10.1016/j.reuma.2012.01.014

117. Washio K, Oka M, Ohno K, Shimizu H, Kawano S, Kunisada M, et al. Case of recurrent Sweet's syndrome in a patient with relapsing polychondritis and myelodysplastic syndrome. J Dermatol. (2012) 39:731-3. doi: 10.1111/j.1346-8138.2011.01403.x

118. Lin J, Zhang Q, Chen M. Subcutaneous histiocytoid Sweet's syndrome in a patient associated with myelodysplastic syndrome-refractory anemia. $J$ Dermatol. (2012) 39:99-101. doi: 10.1111/j.1346-8138.2011.01290.x

119. Calistru AM, Lisboa C, Azevedo F. Paraneoplastic relapsing polychondritis and Sweet syndrome coexisting in a patient with myelodysplasia. Indian J Dermatol Venereol Leprol. (2011) 77:730. doi: 10.4103/0378-6323.86505

120. Diamantino Fda E, Raimundo PM, Fidalgo AI. Sweet's Syndrome and relapsing polychondritis signal myelodysplastic syndrome. Anais Brasileiros de Dermatol. (2011) 86(4 Suppl 1):S173-7.

121. Gill HH, Leung AY, Trendell-Smith NJ, Yeung CK, Liang R. Sweet Syndrome due to Myelodysplastic Syndrome: possible therapeutic role of intravenous immunoglobulin in addition to standard treatment. Adv Hematol. (2010) 2010:328316. doi: 10.1155/2010/328316

122. Xu HH, Xiao $\mathrm{T}$, Gao XH, Chen HD. Ulcerative Sweet syndrome accompanied by interstitial lung disease and myelodysplastic syndrome. Eur J Dermatol. (2009) 19:411-2. 
123. Kato T, Kawana S, Takezaki S-I, Kikuchi S, Futagami A. Case of Sweet's syndrome with extensive necrosis and ulcers accompanied by myelodysplastic syndrome. J Nippon Med Sch. (2008) 75:162-5. doi: 10.1272/jnms.75.162

124. Kawakami T, Kawase A, Takeuchi S, Yoshioka S, Fujimoto N, Tajima S, et al. Sweet syndrome subsequent to relapsing polychondritis and myelodysplastic syndrome in a Japanese patient. Acta Dermato-venereol. (2008) 88:517-9. doi: 10.2340/00015555-0488

125. Khatri ML, Taha M. Sweet's syndrome associated with myelodysplastic syndrome presenting as periorbital cellulitis. Int J Dermatol. (2007) 46:496-9. doi: 10.1111/j.1365-4632.2006.03019.x

126. Garg R, Soud Y, Lal R, Mehta N, Kone BC. Myelodysplastic syndrome manifesting as Sweet's Syndrome and bronchiolitis obliterative organizing pneumonia. Am J Med. (2006) 119:e5-7. doi: 10.1016/j.amjmed.2006.03.032

127. Hattori H, Hoshida S, Yoneda S. Sweet's syndrome associated with recurrent fever in a patient with trisomy 8 myelodysplastic syndrome. Int J Hematol. (2003) 77:383-6. doi: 10.1007/BF02982648

128. Nishie W, Kimura T, Kanagawa M. Sweet's syndrome evolved from recurrent erythema nodosum in a patient with myelodysplastic syndrome. J Dermatol. (2002) 29:91-5. doi: 10.1111/j.1346-8138.2002.tb00172.x

129. Salvador-Osuna C, Fernandez-Mosteirin N, Mayayo P, Delgado P, Giralt M. Choroiditis as systemic manifestation of a Sweet's syndrome associated to myelodysplasia: a case report. Haematologica. (2002) 87:ECR07.

130. Loraas A, Waage A, Lamvik J. Cytokine response pattern in Sweet's syndrome associated with myelodysplasia. Br J Haematol. (1994) 87:669. doi: 10.1111/j.1365-2141.1994.tb08340.x

131. Bajwa RP, Marwaha RK, Garewal G, Rajagopalan M. Acute febrile neutrophilic dermatosis (Sweet's syndrome) in myelodysplastic syndrome. Pediatr Hematol Oncol. (1993) 10:343-6. doi: 10.3109/088800193090 29513

132. Sharpe GR, Leggat HM. A case of Sweet's syndrome and myelodysplasia: response to cyclosporin. $\mathrm{Br} J$ Dermatol. (1992) 127:538-9. doi: 10.1111/j.1365-2133.1992.tb14856.x

133. Barnadas MA, Sitjas D, Brunet S, Puig J, de Moragas JM. Acute febrile neutrophilic dermatosis (Sweet's syndrome) associated with prostate adenocarcinoma and a myelodysplastic syndrome. Int J Dermatol. (1992) 31:647-8. doi: 10.1111/j.1365-4362.1992.tb03987.x

134. Kueh YK, Vijayasingam SM. Severe myelodysplasia with monosomies 5 and 7 presenting with rapidly fatal Sweet's syndrome. Ann Acad Med. (1992) 21:404-7.

135. McNally A, Ibbetson J, Sidhu S. Azathioprine-induced Sweet's syndrome: a case series and review of the literature. Aust J Dermatol. (2017) 58:53-7. doi: 10.1111/ajd.12383

136. Biswas SN, Chakraborty PP, Gantait K, Bar C. Azathioprine-induced bullous Sweet's syndrome: a rare association. BMJ Case Rep. (2016) 2016:1-7. doi: 10.1136/bcr-2016-215192

137. Imhof L, Meier B, Frei P, Kamarachev J, Rogler G, Kolios A, et al. Severe Sweet's Syndrome with Elevated Cutaneous Interleukin-1beta after Azathioprine Exposure: Case Report and Review of the Literature. Dermatology. (2015) 230:293-8. doi: 10.1159/000371879

138. El-Azhary RA, Brunner KL, Gibson LE. Sweet syndrome as a manifestation of azathioprine hypersensitivity. Mayo Clin Proc. (2008) 83:1026-30. doi: $10.4065 / 83.9 .1026$

139. Flores Martin IM, Lopez-Saez MP, Brugaletta Matheus DC, Acosta Ruiz G, Lopez JD, Pagan Aleman JA. Azathioprine-induced Sweet's syndrome. J Invest Allergol Clin Immunol. (2012) 22:66-7.

140. Choonhakarn C, Chaowattanapanit S. Azathioprine-induced Sweet's syndrome and published work review. J Dermatol. (2013) 40:267-71. doi: 10.1111/1346-8138.12081

141. Cyrus N, Stavert R, Mason AR, Ko CJ, Choi JN. Neutrophilic dermatosis after azathioprine exposure. JAMA Dermatol. (2013) 149:592-7. doi: 10.1001/jamadermatol.2013.137

142. Grelle JL, Halloush RA, Khasawneh FA. Azathioprine-induced acute febrile neutrophilic dermatosis (Sweet's syndrome). BMJ Case Rep. (2013) 16:16. doi: 10.1136/bcr-2013-200405

143. Ben Salem C, Larif S, Fathallah N, Slim R, Aounallah A, Sakhri J, et al. A rare case of azathioprine-induced sweet's syndrome in a patient with Crohn's Disease. [Erratum Curr Drug Saf. 2016] Curr Drug Saf. (2016) 10:266-8.
144. Treton X, Joly F, Alves A, Panis Y, Bouhnik Y. Azathioprine-induced Sweet's syndrome in Crohn's disease. Inflamm Bowel Dis. (2008) 14:1757-8. doi: 10.1002/ibd.20518

145. Turow A, Yong TY, Fok JS, Li JY. Azathioprine hypersensitivity presenting as cardiogenic shock and Sweet's syndrome in a patient with microscopic polyangiitis. Int Med. (2012) 51:1889-92. doi: 10.2169/internalmedicine.51.6962

146. Kim MJ, Jang KT, Choe YH. Azathioprine hypersensitivity presenting as sweet syndrome in a child with ulcerative colitis. [Erratum appears in Indian Pediatr. 2012 Jan;49:16]. Indian Pediatr. (2011) 48:969-71.

147. Aleissa M, Nicol P, Godeau M, Tournier E, de Bellissen F, Robic MA, et al. Azathioprine hypersensitivity syndrome: two cases of febrile neutrophilic dermatosis induced by Azathioprine. Case Rep Dermatol. (2017) 9:6-11. doi: 10.1159/000454876

148. Cohen PR. Sweet syndrome and erythema nodosum. Southern Med J. (2007) 100:1057-8. doi: 10.1097/SMJ.0b013e3181514b1b

149. Ginarte M, Toribio J. Association of Sweet syndrome and erythema nodosum. Arch Dermatol. (2000) 136:673-4. doi: 10.1001/archderm.136.5.673-a

150. Waltz KM, Long D, Marks JG Jr., Billingsley EM. Sweet's syndrome and erythema nodosum: the simultaneous occurrence of 2 reactive dermatoses. Arch Dermatol. (1999) 135:62-6. doi: 10.1001/archderm.135.1.62

151. Cohen PR, Holder WR, Rapini RP. Concurrent Sweet's syndrome and erythema nodosum: a report, world literature review and mechanism of pathogenesis. J Rheumatol. (1992) 19:814-20.

152. Wilkinson SM, Heagerty AH, English JS. Acute febrile neutrophilic dermatosis in association with erythema nodosum and sarcoidosis. Clin Exp Dermatol. (1993) 18:47-9. doi: 10.1111/j.1365-2230.1993.tb00966.x

153. Ben-Noun L. Sweet's syndrome associated with erythema nodosum. Aust Family Phys. (1995) 24:1867-9.

154. Gillott TJ, Whallett AJ, Struthers GR, Ilchyshyn A. Concurrent Sweet's syndrome (acute febrile neutrophilic dermatosis), erythema nodosum and sarcoidosis. Clin Exp Dermatol. (1997) 22:54-6.

155. Mazokopakis E, Kalikaki A, Stathopoulos E, Vrentzos G, Papadakis JA. Acute febrile neutrophilic dermatosis (Sweet's syndrome) with erythema nodosum and anterior scleritis. A case report. Int J Dermatol. (2005) 44:1051-3. doi: 10.1111/j.1365-4632.2004.02278.x

156. Tabanlioglu D, Boztepe G, Erkin G, Gokoz O, Karaduman A. Sweet's syndrome and erythema nodosum: a companionship or a spectrum?-a case report with review of the literature. Int J Dermatol. (2010) 49:62-6. doi: 10.1111/j.1365-4632.2009.04093.x

157. Harris T, Henderson MC. Concurrent Sweet's syndrome and erythema nodosum. J Gen Int Med. (2011) 26:214-5. doi: 10.1007/s11606-010-1536-1

158. Grattan CE, Kennedy CT, Glover SC, Mann RJ. Sweet's syndrome and erythema nodosum. Br Med Chirurg J. (1963). (1988) 103:44-5.

159. Spatz SA. Erythema nodosum in Sweet's syndrome. Cutis. (1985) 35:327-30.

160. Gupta N, Chawla B, Venkatesh P, Tandon R. Necrotizing scleritis and peripheral ulcerative keratitis in a case of Sweet's syndrome found culturepositive for Mycobacterium tuberculosis. Ann Trop Med Parasitol. (2008) 102:557-60. doi: 10.1179/136485908X311812

161. Ledoult E, Becquart C, Chanson N, Sobanski V, Remy-Jardin M, Delaporte E, et al. Sweet syndrome and disseminated Mycobacterium tuberculosis infection. Eur J Dermatol. (2016) 26:99-100. doi: 10.1684/ejd.2015.2711

162. Karmakar PS, Sherpa PL, Ray AN, Saha BK, Santra T, Saha S, et al. Sweet's syndrome: a very rare association with pulmonary tuberculosis. J Infect Dev Count. (2013) 7:417-20. doi: 10.3855/jidc.2606

163. Singh RK. Acute febrile neutrophilic dermatosis following tuberculous infection. J Assoc Phys India. (2002) 50:1322-3.

164. Mrabet D, Saadi F, Zaraa I, Chelly I, Sahli H, Ben Osmane A, et al. Sweet's syndrome in a patient with rheumatoid arthritis, Sjogren's syndrome and lymph node tuberculosis. BMJ Case Rep. (2011) 2011:1-4. doi: 10.1136/bcr.07.2010.3137

165. Serirat O, Thaipisuttikul Y. Sweet's syndrome associated with Mycobacterium tuberculosis and cervical cancer: a case report. J Med Assoc Thailand. (2011) 94 (Suppl. 2):S119-22.

166. Chauhan S. An extremely rare association of Sweet's syndrome with active pulmonary tuberculosis. Ind J Tuberculosis. (2018) 65:87-90. doi: $10.1016 /$ j.ijtb.2017.04.005 
167. Brodkin RH, Schwartz RA. Sweet's syndrome with myelofibrosis and leukemia: partial response to interferon. Dermatology. (1995) 190:160-3. doi: $10.1159 / 000246669$

168. Nakanishi K, Kinjo M. Mimicker of necrotising fasciitis with systemic inflammatory response syndrome: recurrent necrotising Sweet's syndrome associated with chronic myelogenous leukaemia. BMJ Case Rep. (2016) 31:31. doi: 10.1136/bcr-2016-214461

169. dos Santos VM, Nery NS, Bettarello G, Neiman IM, de Brito FC, Souza CF. Photoclinic. Bullous Sweet syndrome in chronic myeloid leukemia. Arch Iranian Med. (2010) 13:561-2.

170. Liu D, Seiter K, Mathews T, Madahar CJ, Ahmed T. Sweet's syndrome with CML cell infiltration of the skin in a patient with chronic-phase CML while taking Imatinib Mesylate. Leukemia Res. (2004) 28 (Suppl. 1):S61-3. doi: 10.1016/S0145-2126(03)00257-1

171. Fernandez-Jimenez MC, Herraez R, Ojeda E, Hernandez-Navarro F. Sweet's syndrome and accelerated phase of chronic myelogenous leukemia. Ann Hematol. (2000) 79:585-7. doi: 10.1007/s002770000191

172. Urano Y, Miyaoka Y, Kosaka M, Kabe K, Uchida N, Arase S. Sweet's syndrome associated with chronic myelogenous leukemia: demonstration of leukemic cells within a skin lesion. J Am Acad Dermatol. (1999) 40(2 Pt 1):275-9. doi: 10.1016/S0190-9622(99)70206-9

173. Kannan R, Dutta TK, Goel A, Garg BR, Venkateswaran S, Ratnakar C. Sweet syndrome in chronic myeloid leukaemia. Postgr Med J. (1995) 71:383. doi: $10.1136 /$ pgmj.71.836.383-a

174. Feliu E, Cervantes F, Ferrando J, Puig S, Mascaro JM, Rozman C. Neutrophilic pustulosis associated with chronic myeloid leukemia: a special form of Sweet's syndrome. Report of two cases. Acta Haematol. (1992) 88(2-3):154-7. doi: 10.1159/000204674

175. Gonzalez-Castro U, Julia A, Pedragosa R, Bueno J, Vidal J, Castells A. Sweet syndrome in chronic myelogenous leukemia. Int J Dermatol. (1991) 30:648-50. doi: 10.1111/j.1365-4362.1991.tb03492.x

176. Mijovic A, Mijovic A, Medenica L, Rolovic Z. Sweet's syndrome in chronic phase of chronic myeloid leukaemia. Eur J Cancer. (1991) 27:1336. doi: 10.1016/0277-5379(91)90115-T

177. Pertusi RM, Forman MD, Brown AC. Sweet's syndrome after splenic irradiation for chronic myelogenous leukemia. J Am Osteopathic Assoc. (1996) 96:366-7. doi: 10.7556/jaoa.1996.96.6.366

178. Astudillo L, Loche F, Reynish W, Rigal-Huguet F, Lamant L, Pris J. Sweet's syndrome associated with retinoic acid syndrome in a patient with promyelocytic leukemia. Ann Hematol. (2002) 81:111-4. doi: 10.1007/s00277-001-0416-5

179. Shirono K, Kiyofuji C, Tsuda H. Sweet's syndrome in a patient with acute promyelocytic leukemia during treatment with all-trans retinoic acid. Int $J$ Hematol. (1995) 62:183-7. doi: 10.1016/0925-5710(95)00403-F

180. Jagdeo J, Campbell R, Long T, Muglia J, Telang G, Robinson-Bostom L. Sweet's syndrome-like neutrophilic lobular panniculitis associated with all-trans-retinoic acid chemotherapy in a patient with acute promyelocytic leukemia. J Am Acad Dermatol. (2007) 56:690-3. doi: 10.1016/j.jaad.2006.08.011

181. Piette WW, Trapp JF, O’Donnell MJ, Argenyi Z, Talbot EA, Burns CP. Acute neutrophilic dermatosis with myeloblastic infiltrate in a leukemia patient receiving all-trans-retinoic acid therapy. J Am Acad Dermatol. (1994) 30(2 Pt 2):293-7. doi: 10.1016/S0190-9622(94)70026-5

182. Cox NH, O'Brien HA. Sweet's syndrome associated with trans-retinoic acid treatment in acute promyelocytic leukaemia. Clin Exp Dermatol. (1994) 19:51-2. doi: 10.1111/j.1365-2230.1994.tb01115.x

183. Christ E, Linka A, Jacky E, Speich R, Marincek B, Schaffner A. Sweet's syndrome involoving the musculoskeletal system during treatment of promyelocytic leukemia with all-trans retinoic acid. Leukemia. (1996) 10:731-4.

184. Arun B, Berberian B, Azumi N, Frankel SR, Luksenburg H, Freter C. Sweet's syndrome during treatment with all-trans retinoic acid in a patient with acute promyelocytic leukemia. Leukemia Lymphoma. (1998) 31(5-6):613-5. doi: 10.3109/10428199809057622

185. Levi I, Raanani P, Shalmon B, Schiby-Brilliant R, Ben-Bassat I. Acute neutrophilic dermatosis induced by all-trans-retinoic acid treatment for acute promyelocytic leukemia. Leukemia Lymphoma. (1999) 34(3-4):401-4. doi: $10.3109 / 10428199909050966$
186. Takada S, Matumoto K, Sakura T, Shiozaki H, Miyawaki S. Sweet's syndrome followed by retinoic acid syndrome during the treatment of acute promyelocytic leukemia with all-trans retinoic acid. Int J Hematol. (1999) 70:26-9.

187. Park CJ, Bae YD, Choi JY, Heo PS, Lee KS, Park YS, et al. Sweet's syndrome during the treatment of acute promyelocytic leukemia with all-trans retinoic acid. Korean J Int Med. (2001) 16:218-21. doi: 10.3904/kjim.2001.16.3.218

188. Tomas JF, Escudero A, Fernandez-Ranada JM. All-trans retinoic acid treatment and Sweet syndrome. Leukemia. (1994) 8:1596.

189. Cuende Quintana E, Gomez Rz de Mendarozqueta M, Gorospe Arrazuria MA, Saracibar Oyon N, Atares Pueyo B, Pena MV, et al. Concurrent Sweet's syndrome and Lofgren's syndrome. J Rheumatol. (1996) 23:1995-8.

190. Dadban A, Hirschi S, Sanchez M, Lagrange B. Association of Sweet's syndrome and acute sarcoidosis: report of a case and review of the literature. Clin Expe Dermatol. (2009) 34:189-91. doi: 10.1111/j.1365-2230.2008.02813.x

191. Pouchot J, Bourgeois-Droin C, Vinceneu P, Barge J, Brun P, Granier F, et al. Sweet's syndrome and mediastinal lymphadenopathy due to sarcoidosis: three cases of a new association. Arch Dermatol. (1993) 129:1062-4. doi: 10.1001/archderm.1993.01680290138029

192. Ganeshakrishnan KT, Ott GY, Barker A, Cobanoglu A. Sweet's syndrome and associated sarcoidosis-a rare clinical case. Thoracic Cardiovasc Surg. (1997) 45:247-8. doi: 10.1055/s-2007-1013737

193. Stuveling EM, Fedder G, Bruns HM, Vos H, Eggelmeijer F. The association of Sweet's syndrome with sarcoidosis. Netherlands J Med. (2001) 59:31-4. doi: 10.1016/S0300-2977(01)00125-5

194. Baz K, Yazici AC, Kaya TI, Ikizoglu G, Ulubas B, Apa DD, et al. Neutrophilic dermatosis of the hands (localized Sweet's syndrome) in association with chronic hepatitis C and sarcoidosis. Clin Exp Dermatol. (2003) 28:377-9. doi: 10.1046/j.1365-2230.2003.01299.x

195. Gkrouzman E, Chirch L, Lakshminarayanan S. Drug-induced sweet syndrome in a man with sarcoidosis: are there any common mechanisms of pathogenesis? J Clin Rheumatol. (2018). doi: 10.1097/00124743-900000000-99320. [Epub ahead of print].

196. Saliba WR, Habib GS, Elias M. Sweet's syndrome and sarcoidosis. Eur J Int Med. (2005) 16:545-50. doi: 10.1016/j.ejim.2005.06.017

197. Kyrmizakis DE, Drivas E, Kruger-Krasagakis S, Hajiioannou I, Karatzanis A, Velegrakis GA. Acute rhinosinusitis associated with Sweet's syndrome. J Otolaryngol. (2006) 35:144-7. doi: 10.2310/7070.2005.5011

198. Miyamura T, Kajihara I, Makino K, Ihn H. Sweet's syndrome with laryngoparalysis due to laryngitis. J Dermatol. (2018) 45:e295-6. doi: $10.1111 / 1346-8138.14351$

199. Lallas A, Tzellos TG, Papageorgiou M, Mandekou-Lefaki I. Sweet's syndrome associated with upper respiratory tract streptococcal infection: "wait-andsee" strategy or anecdotal use of corticosteroids? Hippokratia. (2011) 15:283.

200. Volpe M. Sweet Syndrome Associated with Upper Respiratory Infection and Amoxicillin Use. Cureus. (2016) 8:e568. doi: 10.7759/cureus.568

201. Gaopande VL, Joshi SS, Joshi AR. Acute promyelocytic leukemia-associated Sweet's syndrome mimicking an axillary abscess: a case report with review of literature. Diagn Cytopathol. (2015) 43:1007-10. doi: 10.1002/dc.23367

202. Gilmour E, Chalmers RJ, Rowlands DJ. Drug-induced Sweet's syndrome (acute febrile neutrophilic dermatosis) associated with hydralazine. $\mathrm{Br} \mathrm{J}$ Dermatol. (1995) 133:490-1. doi: 10.1111/j.1365-2133.1995.tb02686.x

203. Cartee TV, Chen SC. Sweet syndrome associated with hydralazine-induced lupus erythematosus. Cutis. (2012) 89:121-4.

204. Juanola X, Nolla JM, Servitje O, Valverde J. Hydralazine induced lupus and Sweet's syndrome. J Rheumatol. (1991) 18:948.

205. Sequeira W, Polisky RB, Alrenga DP. Neutrophilic dermatosis (Sweet's syndrome). Association with a hydralazine-induced lupus syndrome. Am J Med. (1986) 81:558-60. doi: 10.1016/0002-9343(86)90316-5

206. Servitje O, Ribera M, Juanola X, Rodriguez-Moreno J. Acute neutrophilic dermatosis associated with hydralazine-induced lupus. Arch Dermatol. (1987) 123:1435-6. doi: 10.1001/archderm.1987.01660350029008

207. Ramsey-Goldman R, Franz T, Solano FX, Medsger TA, Jr. Hydralazine induced lupus and Sweet's syndrome. Report and review of the literature. $J$ Rheumatol. (1990) 17:682-4.

208. Miyauchi T, Nishie W, Sakata M, Osawa R, Noguchi A, Shimizu H. Sweet syndrome-like eruption with prominent dermal leukocytoclasis 
associated with systemic lupus erythematosus. J Dermatol. (2015) 42:442-3. doi: 10.1111/1346-8138.12802

209. Quinn N, MacMahon J, Irvine AD, Lowry C. Sweet syndrome revealing systemic lupus erythematosus. Irish Med J. (2015) 108:59-60.

210. Tsuji H, Yoshifuji H, Nakashima R, Imura Y, Yukawa N, Ohmura K, et al. Sweet's syndrome associated with systemic lupus erythematosus: a case report and review of the literature. J Dermatol. (2013) 40:641-8. doi: 10.1111/1346-8138.12184

211. Choi JW, Chung KY. Sweet's syndrome with systemic lupus erythematosus and herpes zoster. Br J Dermatol. (1999) 140:1174-5.

212. Hou TY, Chang DM, Gao HW, Chen CH, Chen HC, Lai JH. Sweet's syndrome as an initial presentation in systemic lupus erythematosus: a case report and review of the literature. Lupus. (2005) 14:399-402. doi: 10.1191/0961203305lu2083cr

213. Burnham JM, Cron RQ. Sweet syndrome as an initial presentation in a child with systemic lupus erythematosus. Lupus. (2005) 14:974-5. doi: 10.1191/0961203305lu2236xx

214. Gollol-Raju N, Bravin M, Crittenden D. Sweet's syndrome and systemic lupus erythematosus. Lupus. (2009) 18:377-8. doi: 10.1177/0961203308100046

215. Gheorghe L, Cotruta B, Trifu V, Cotruta C, Becheanu G, Gheorghe C. Druginduced Sweet's syndrome secondary to hepatitis $\mathrm{C}$ antiviral therapy. Int $J$ Dermatol. (2008) 47:957-9. doi: 10.1111/j.1365-4632.2008.03724.x

216. Guo D, Parsons LM. Sweet syndrome in a patient with chronic hepatitis C. J Cutaneous Med Surg. (2014) 18:436-8. doi: 10.2310/7750.2014.13211

217. Tewari A, Chandrakumar A, Macdonald D, Staughton R, Bunker CB. Sweet syndrome preceding a carcinoid lung tumor and multiple myeloma. Cutis. (2013) 92:E1.

218. Kim JS, Roh HS, Lee JW, Lee MW, Yu HJ. Distinct variant of Sweet's syndrome: bortezomib-induced histiocytoid Sweet's syndrome in a patient with multiple myeloma. Int J Dermatol.. (2012) 51:1491-3. doi: 10.1111/j.1365-4632.2011.05141.x

219. Belhadjali H, Chaabane S, Njim L, Youssef M, Zakhama A, Zili J. Sweet's syndrome associated with multiple myeloma. Acta Dermatovenerol Alpina Panonica Adriatica. (2008) 17:31-3.

220. Torralbo A, Herrero JA, del-Rio E, Sanchez-Yus E, Barrientos A. Sweet's syndrome associated with multiple myeloma. Int J Dermatol. (1992) 31:297-8. doi: 10.1111/j.1365-4362.1992.tb03581.x

221. Colovic MD, Jankovic GM, Novak AZ, Strahinja RM, Colovic NR. Sweet's syndrome associated with paracentric inversion of chromosome $3 \mathrm{q}$ in a patient with multiple myeloma. Eur J Haematol. (1996) 57:188-9. doi: 10.1111/j.1600-0609.1996.tb01361.x

222. Bayer-Garner IB, Cottler-Fox M, Smoller BR. Sweet syndrome in multiple myeloma: a series of six cases. J Cutaneous Pathol. (2003) 30:261-4. doi: 10.1046/j.0303-6987.2002.029.x

223. Borbujo J, de Lucas R, Manzano R, Casado M. [Sweet's syndrome and multiple myeloma]. Med Clin. (1996) 106:354-5.

224. Breier F, Hobisch G, Groz S. [Sweet syndrome. Acute neutrophilic dermatosis in multiple myeloma]. Der Hautarzt; Zeitschrift fur Dermatologie Venerologie Verwandte Gebiete. (1993) 44:229-31.

225. Fauconnier SA, Leclercq A, Thomas T, Labeille B, Marotte H. Neutrophil dermatosis of the dorsal hands with multiple myeloma. Joint Bone Spine. (2016) 83:227. doi: 10.1016/j.jbspin.2015.03.011

226. Tazi I, Nafil H, Mahmal L. Immunoglobulin a multiple myeloma associated with sweet syndrome. J Cancer Res Therap. (2012) 8:652-3. doi: 10.4103/0973-1482.106591

227. Llamas-Velasco M, Concha-Garzon MJ, Fraga J, Aragues M. Histiocytoid sweet syndrome related to bortezomib: a mimicker of cutaneous infiltration by myeloma. Indian J Dermatol, Venereol Leprol. (2015) 81:305-6. doi: 10.4103/0378-6323.152743

228. Knoops L, Jacquemain A, Tennstedt D, Theate I, Ferrant A, Van den Neste E. Bortezomib-induced Sweet syndrome. Br J Haematol. (2005) 131:142. doi: 10.1111/j.1365-2141.2005.05636.x

229. Van Regenmortel N, Van de Voorde K, De Raeve H, Rombouts S, Van de Velde A, Lambert J, et al. Bortezomib-induced Sweet's syndrome. Haematologica. (2005) 90(12 Suppl):ECR43.

230. Murase JE, Wu JJ, Theate I, Cole GW, Barr RJ, Dyson SW. Bortezomibinduced histiocytoid Sweet syndrome. J Am Acad Dermatol. (2009) 60:496-7. doi: 10.1016/j.jaad.2008.09.007
231. Zobniw CM, Saad SA, Kostoff D, Barthel BG. Bortezomib-induced Sweet's syndrome confirmed by rechallenge. Pharmacotherapy. (2014) 34:e18-21. doi: 10.1002/phar.1383

232. Arima Y, Namiki T, Ueno M, Kato K, Tokoro S, Takayama K, et al. Histiocytoid Sweet syndrome: a novel association with relapsing polychondritis. $\mathrm{Br} \quad J$ Dermatol. (2016) 174:691-4. doi: 10.1111/ bjd. 14229

233. Vestergaard C, Soelvsten H, Ramsing M, Hansen ES, Deleuran M. Concomitant Sweet's syndrome and relapsing polychondritis. Acta DermatoVenereol. (2007) 87:426-7. doi: 10.2340/00015555-0263

234. Fujimoto N, Tajima S, Ishibashi A, Ura-Ishikou A, Manaka I. Acute febrile neutrophilic dermatosis (Sweet's syndrome) in a patient with relapsing polychondritis. $\mathrm{Br} \quad J$ Dermatol. (1998) 139:930-1. doi: $10.1046 / j .1365-2133.1998 .02536 . x$

235. Astudillo L, Launay F, Lamant L, Sailler L, Bazex J, Couret B, et al. Sweet's syndrome revealing relapsing polychondritis. Int J Dermatol. (2004) 43:720-2. doi: 10.1111/j.1365-4632.2004.02198.x

236. Cohen PR. Sweet's syndrome and relapsing polychondritis: is their appearance in the same patient a coincidental occurrence or a bona fide association of these conditions? Int J Dermatol. (2004) 43:772-7. doi: $10.1111 /$ j.1365-4632.2004.02197.x

237. Vano-Galvan S, Perez-Carmona L, Moreno C, Jaen P. Sweet syndrome after gastrointestinal tract infection. Israel Med Assoc J. (2010) 12:128.

238. Papadatos SS, Zissis C, Deligiannis G, Mylonas S. Enteritis-associated acute febrile neutrophilic dermatosis with acute monoarthritis. Folia Med. (2017) 59:477-80. doi: 10.1515/folmed-2017-0057

239. Pai S, Rytina E, Sterling J, Karas JA, Aliyu SH. Campylobacter gastroenteritis associated with Sweet's syndrome. J Med Microbiol. (2012) 61(Pt 10):1473-5. doi: 10.1099/jmm.0.044412-0

240. Elsner P, Hartmann AA, Lechner W. Sweet's syndrome associated with Yersinia enterocolitica infection. Dermatologica. (1986) 173:85-9. doi: $10.1159 / 000249224$

241. Florez A, Sanchez-Aguilar D, Roson E, Prieto A, Van den Eyden A, Toribio J. Sweet's syndrome associated with salmonella enteritidis infection. Clin Exp Dermatol. (1999) 24:239-40. doi: 10.1046/j.1365-2230.1999. 00465.x

242. Chang SS, Chau WK, Liu MT, Ho CH. Acute febrile neutrophilic dermatosis (Sweet's syndrome) in hairy cell leukemia: a case report. Chung Hua i Hsueh Tsa Chih. (1999) 62:467-71.

243. Levy RM, Junkins-Hopkins JM, Turchi JJ, James WD. Sweet syndrome as the presenting symptom of relapsed hairy cell leukemia. Arch Dermatol. (2002) 138:1551-4. doi: 10.1001/archderm.138.12.1551

244. Ventura F, Rocha J, Pereira T, Marques H, Pardal F, Brito C. Sweet syndrome as the presenting symptom of hairy cell leukemia. Dermatol Online J. (2009) 15:12.

245. Ozdogu H, Yeral M, Boga C. An Unusual Giant Leg Ulcer as a Rare Presentation of Sweet's Syndrome in a patient with hairy cell leukemia successfully managed by splenectomy. Turkish J Haematol. (2017) 34:270-1. doi: 10.4274/tjh.2016.0416

246. Alkayem M, Cheng W. A case report of hairy cell leukemia presenting concomitantly with sweet syndrome. Case Rep Med. (2014) 2014:823286. doi: $10.1155 / 2014 / 823286$

247. Dalri P, Boi S, Cristofolini M, Piscioli F, Rubertelli M. Sweet syndrome: presenting symptom of hairy cell leukemia with fatal infection by pneumocystis carinii. Haematologica. (1982) 67:765-8.

248. Gisser SD. Acute febrile neutrophilic dermatosis (Sweet's syndrome) in a patient with hairy-cell leukemia. Am J Dermatopathol. (1983) 5:283-8. doi: 10.1097/00000372-198306000-00016

249. Fischer G, Commens C, Bradstock K. Sweet's syndrome in hairy cell leukemia. J Am Acad Dermatol. (1989) 21(3 Pt 1):573-4. doi: 10.1016/S0190-9622(89)80232-4

250. Walker DC, Cohen PR. Trimethoprim-sulfamethoxazole-associated acute febrile neutrophilic dermatosis: case report and review of drug-induced Sweet's syndrome. J Am Acad Dermatol. (1996) 34(5 Pt 2):918-23. doi: 10.1016/S0190-9622(96)90080-8

251. Kluger N, Marque M, Stoebner PE, Dandurand M, Meunier L. Possible druginduced Sweet's syndrome due to trimethoprim-sulfamethoxazole. Acta Dermato-Venereol. (2008) 88:637-8. doi: 10.2340/00015555-0516 
252. Azfar RS, Cohn J, Schaffer A, Kim EJ. Trimethoprim sulfamethoxazoleinduced sweet syndrome. Arch Dermatol. (2009) 145:215-6. doi: 10.1001/archderm.145.2.215

253. Khaled A, Kharfi M, Fazaa B, Kourda M, Bouaziz A, Kastalli S, et al. A first case of trimethoprim-sulfamethoxazole induced Sweet's syndrome in a child. Pediatr Dermatol. (2009) 26:744-6. doi: 10.1111/j.1525-1470.2009. 00868.x

254. Su WP, Liu HN. Diagnostic criteria for Sweet's syndrome. Cutis. (1986) $37: 167-74$

255. de Boysson H, Martin Silva N, de Moreuil C, Neel A, de Menthon M, Meyer $\mathrm{O}$, et al. Neutrophilic dermatoses in antineutrophil cytoplasmic antibodyassociated vasculitis: a french multicenter Study of 17 cases and literature review. Medicine. (2016) 95:e2957. doi: 10.1097/MD.0000000000002957

256. Sato M, Kawamura T, Hase S, Katsumata S, Oshika T. A case of bilateral retinal vasculitis associated with Sweet syndrome. Retina. (2005) 25:800-2. doi: 10.1097/00006982-200509000-00024

257. Saussine A, Gueguen A, de Menthon M, Maisonobe T, Battistella M, Serrato $\mathrm{T}$, et al. Sweet syndrome revealing microscopic polyangiitis. Rheumatology. (2012) 51:1916-7. doi: 10.1093/rheumatology/kes060

258. Miraliakbari HM, McEarchen J, Prasad B. Sweet's syndrome in a patient with Wegener's granulomatosis and ESRD. BMJ Case Rep. (2012) 27:27. doi: $10.1136 /$ bcr.08.2010.3231

259. Campos LM, Castellanos AL, Afiune JY, Kiss MH, Silva CA. Takayasu's arteritis with aortic aneurysm associated with Sweet's syndrome in childhood. Ann Rheum Dis. (2005) 64:168-9. doi: 10.1136/ard.2003.006353

260. Ma EH, Akikusa JD, MacGregor D, Ng J, Su JC. Sweet's syndrome with postinflammatory elastolysis and Takayasu arteritis in a child: a case report and literature review. Pediatr Dermatol. (2012) 29:645-50. doi: 10.1111/j.1525-1470.2011.01597.x

261. Neild GH, Silva C, Afonso N, Carreira A, Campos M. Azathioprine-induced Sweet syndrome in ANCA-associated vasculitis. Clin Kidn J. (2013) 6:657-8. doi: $10.1093 / \mathrm{ckj} / \mathrm{sft} 134$

262. Delaporte E, Gaveau DJ, Piette FA, Bergoend HA. Acute febrile neutrophilic dermatosis (Sweet's syndrome). Association with rheumatoid vasculitis. Arch Dermatol. (1989) 125:1101-4. doi: 10.1001/archderm.1989.016702000 77013

263. Endo Y, Tanioka M, Tanizaki H, Mori M, Kawabata H, Miyachi Y. Bullous Variant of Sweet's Syndrome after Herpes Zoster Virus Infection. Case Rep Dermatol. (2011) 3:259-62. doi: 10.1159/000334833

264. Smith CR, Williams P. Sweet's syndrome in a patient with chronic lymphocytic leukaemia. BMJ Case Rep. (2017) doi: 10.1136/bcr-2017-220317. [Epub ahead of print]

265. Cholongitas E, Pipili C, Dasenaki M, Kaklamanis L. Piperacillin/tazobactaminduced Sweet syndrome in a patient with chronic lymphocytic leukemia and autoimmune cholangitis. Am J Dermatopathol. (2008) 30:203-4. doi: 10.1097/DAD.0b013e318165db4a

266. Mineo F, Pezzarossa E, Baroni MC, Alinovi A, Crotti G, Delsignore R. Sweet's syndrome in chronic lymphocytic leukemia. Acta Bio-Medica de L'Ateneo Parmense. (1984) 55(3-4):197-8.

267. Ilchyshyn A, Smith AG, Phaure TA. Sweet's syndrome associated with chronic lymphatic leukaemia. Clin Exp Dermatol. (1987) 12:277-9. doi: 10.1111/j.1365-2230.1987.tb01920.x

268. Visani G, Patrizi A, Colombini R, Balducci A, Cenacchi A, Gamberi B. Sweet's syndrome and chronic lymphocytic leukemia associated with scirrhous breast cancer. A case report. Haematologica. (1990) 75:173-5.

269. Usul Afsar C, Paydas S, Gunaldi M, Bozkurt Duman B, Ercolak $\mathrm{V}$, Zorludemir S, et al. Sweet syndrome in a patient with chronic lymphocytic leukemia/small lymphocytic lymphoma: curious lymphocyte/neutrophil fluctuations. Turkish J Haematol. (2013) 30:413-5. doi: 10.4274/Tjh.2012.0055

270. Mensing H, Kowalzick L. Acute febrile neutrophilic dermatosis (Sweet's syndrome) caused by minocycline. Dermatologica. (1991) 182:43-6. doi: $10.1159 / 000247736$

271. Thibault MJ, Billick RC, Srolovitz H. Minocycline-induced Sweet's syndrome. J Am Acad Dermatol. (1992) 27(5 Pt 2):801-4. doi: 10.1016/0190-9622(92)70251-A
272. Kalai C, Brand R, Yu L. Minocycline-induced Sweet syndrome (acute febrile neutrophilic dermatosis). J Am Acad Dermatol. (2012) 67:e289-91. doi: 10.1016/j.jaad.2012.07.005

273. Khan Durani B, Jappe U. Drug-induced Sweet's syndrome in acne caused by different tetracyclines: case report and review of the literature. $\mathrm{Br} J$ Dermatol. (2002) 147:558-62. doi: 10.1046/j.1365-2133.2002.04817.x

274. Jamet A, Lagarce L, Le Clec'h C, Croue A, Hoareau F, Diquet B, et al. Doxycycline-induced Sweet's syndrome. Eur J Dermatol . (2008) 18:595-6.

275. Davies MG, Hastings A. Sweet's syndrome progressing to pyoderma gangrenosum-a spectrum of neutrophilic skin disease in association with cryptogenic cirrhosis. Clin Exp Dermatol. (1991) 16:279-82. doi: 10.1111/j.1365-2230.1991.tb00375.x

276. Burton JL. Sweet's syndrome, pyoderma gangrenosum and acute leukaemia. Br J Dermatol. (1980) 102:239. doi: 10.1111/j.1365-2133.1980.tb05700.x

277. Oskay T, Karademir A, Kutluay L. Sweet's syndrome associated with cytomegalovirus infection. Int J Dermatol. (2004) 43:57-9. doi: 10.1111/j.1365-4632.2004.01812.x

278. Xenophontos E, Ioannou A, Constantinides T, Papanicolaou E. Sweet syndrome on a patient with autoimmune hepatitis on azathioprine and CMV infection. Oxford Med Case Rep. (2016) 2016:24-7. doi: 10.1093/omcr/omw004

279. Inanc SE, Altum M, Onat H, Erseven G. Sweet's syndrome and Hodgkin's disease. Acta Oncol. (1994) 33:574-5.

280. Suvajdzic N, Dimcic Z, Cvijetic O, Colovic M. Sweet's syndrome associated with Hodgkin's disease. Haematologia. (1998) 29:157-8.

281. Miranda CV, Filgueiras Fde M, Obadia DL, Gripp AC, Alves Mde F. Sweet's Syndrome associated with Hodgkin's disease: case report. Anais Brasileiros de Dermatol. (2011) 86:1016-8. doi: 10.1590/S0365-05962011000500025

282. Carvalho R, Fernandes C, Afonso A, Cardoso J. Drug-induced Sweet's syndrome by aceclofenac. Cutaneous Ocular Toxicol. (2011) 30:315-6. doi: 10.3109/15569527.2011.573833

283. Fye KH, Crowley E, Berger TG, LeBoit PE, Connolly MK. Celecoxibinduced Sweet's syndrome. J Am Acad Dermatol. (2001) 45:300-2. doi: $10.1067 / \mathrm{mjd} .2001 .114587$

284. Oh EH, Shin JM, Hong JH, Kim JS, Ro YS, Ko JY. Drug-induced bullous Sweet's syndrome by celecoxib. J Dermatol. (2016) 43:1092-3. doi: 10.1111/1346-8138.13337

285. Rosmaninho A, Lobo I, Selores M. Sweet's syndrome associated with the intake of a selective cyclooxygenase-2 (COX-2) inhibitor. Cutaneous Ocular Toxicol. (2011) 30:298-301. doi: 10.3109/15569527.2011.579929

286. Gupta SK, Bajpai M, Uraiya D. Diclofenac-induced Sweet's syndrome. Indian J Dermatol. (2015) 60:424. doi: 10.4103/0019-5154.160548

287. Nurre LD, Rabalais GP, Callen JP. Neutrophilic dermatosis-associated sterile chronic multifocal osteomyelitis in pediatric patients: case report and review. Pediatr Dermatol. (1999) 16:214-6. doi: 10.1046/j.1525-1470.1999.00058.x

288. Arndt JH. Sweet's syndrome and chronic recurrent multifocal osteomyelitis. Am J Dis Child. (1960). (1987) 141:721.

289. Majeed HA, Kalaawi M, Mohanty D, Teebi AS, Tunjekar MF, al-Gharbawy F, et al. Congenital dyserythropoietic anemia and chronic recurrent multifocal osteomyelitis in three related children and the association with Sweet syndrome in two siblings. J Pediatr. (1989) 115(5 Pt 1):730-4. doi: 10.1016/S0022-3476(89)80650-X

290. Tan E, Yosipovitch G, Giam YC, Tan SH. Bullous Sweet's syndrome associated with acute hepatitis B infection: a new association. Br J Dermatol. (2000) 143:914-6. doi: 10.1046/j.1365-2133.2000.03810.x

291. Park CW, Kim YJ, Seo HJ, Lee KI, Jang BK, Hwang JS, et al. A case of Sweet's syndrome in a patient with liver cirrhosis caused by chronic hepatitis B. Korean J Gastroenterol. (2012) 59:441-4. doi: 10.4166/kjg.2012.59.6.441

292. Woodrow SL, Munn SE, Basarab T, Russel Jones R. Sweet's syndrome in association with non-Hodgkin's lymphoma. Clin Exp Dermatol. (1996) 21:357-9. doi: 10.1111/j.1365-2230.1996.tb00123.x

293. Vestey JP, Judge M. Sweet's syndrome and non-Hodgkin's lymphoma: the first report of this association. Acta Dermato-Venereol. (1985) 65:564-6.

294. Tintle S, Patel V, Ruskin A, Halasz C. Azacitidine: a new medication associated with Sweet syndrome. J Am Acad Dermatol. (2011) 64:e77-9. doi: 10.1016/j.jaad.2010.06.032 
295. Trickett HB, Cumpston A, Craig M. Azacitidine-associated Sweet's syndrome. Am J Health Syst Pharm. (2012) 69:869-71. doi: 10.2146/ajhp110523

296. Kawano H, Suzuki T, Ishii S, Wakahashi K, Kawano Y, Sada A, et al. Recurrence of abdominal large-vessel vasculitis and development of severe Sweet syndrome after a single cycle of 5-azacytidine in a patient with myelodysplastic syndrome. Eur J Haematol. (2014) 92:362-4. doi: 10.1111/ejh.12249

297. Pang A, Tan KB, Aw D, Hsieh WS, Goh BC, Lee SC. A case of Sweet's syndrome due to 5-Azacytidine and vorinostat in a patient with NK/T cell lymphoma. Cutaneous Ocular Toxicol. (2012) 31:64-6. doi: 10.3109/15569527.2011.602034

298. Bonazza S, Dalton B, Hardin J, Metelitsa A. Histiocytoid Variant of Sweet Syndrome Associated with Azacitidine and Recurrence upon Rechallenge. Can J Hospital Pharm. (2015) 68:339-41. doi: 10.4212/cjhp.v68i4.1475

299. Tiwari SM, Caccetta T, Kumarasinghe SP, Harvey N. Azacitidine-induced Sweet syndrome: Two unusual clinical presentations. Aust J Dermatol. (2018) 59:e224-e5. doi: 10.1111/ajd.12727

300. Miura T, Ohtsuka M, Yamamoto T. Sweet's syndrome-like eruption in association with the exacerbation of Behcet's disease after the Great East Japan Earthquake. Actas Dermo-Sifiliogr. (2017) 108:70-2. doi: 10.1016/j.ad.2016.07.006

301. Karadogan SK, Baskan EB, Alkan G, Saricaoglu H, Tunali S. Generalized Sweet syndrome lesions associated with Behcet disease: a true association or simply co-morbidity? Am J Clin Dermatol. (2009) 10:331-5. doi: 10.2165/11310790-000000000-00000

302. Hassikou H, Tabache F, Baaj M, Safi S, Hadri L. Sweet's syndrome in Behcet's disease. Joint Bone Spine. (2007) 74:495-6. doi: 10.1016/j.jbspin.2006.11.022

303. Oguz O, Serdaroglu S, Tuzun Y, Erdogan N, Yazici H, Savaskan H. Acute febrile neutrophilic dermatosis (Sweet's syndrome) associated with Behcet's disease. Int J Dermatol. (1992) 31:645-6. doi: $10.1111 / j .1365-4362.1992 . t b 03986 . x$

304. Lee MS, Barnetson RS. Sweet's syndrome associated with Behcet's disease. Aust J Dermatol. (1996) 37:99-101. doi: 10.1111/j.1440-0960.1996.tb01015.x

305. Wu F, Luo X, Yuan G. Sweet's syndrome representing a flare of Behcet's disease. Clin Exp Rheumatol. (2009) 27(2 Suppl 53):S88-90.

306. Fortna RR, Toporcer M, Elder DE, Junkins-Hopkins JM. A case of sweet syndrome with spleen and lymph node involvement preceded by parvovirus B19 infection, and a review of the literature on extracutaneous sweet syndrome. Am J Dermatopathol. (2010) 32:621-7. doi: 10.1097/DAD.0b013e3181ce5933

307. Gutierrez-Gonzalez E, Alvarez-Perez A, Sanchez-Aguilar D, Toribio J. Sweet's syndrome and acute parvovirus B19 infection. Int J Dermatol. (2013) 52:1611-3. doi: 10.1111/j.1365-4632.2012.05467.x

308. Kaur S, Bery A, Garg B, Sood N. Sweet's syndrome associated with chronic neutrophilic leukemia. Indian J Dermatol Venereol Leprol. (2015) 81:203-6. doi: 10.4103/0378-6323.152304

309. Pedrosa AF, Morais P, Nogueira A, Pardal J, Azevedo F. Sweet's syndrome triggered by pneumococcal vaccination. Cutaneous Ocular Toxicol. (2013) 32:260-1. doi: 10.3109/15569527.2012.759960

310. Jovanovic M, Poljacki M, Vujanovic L, Duran V. Acute febrile neutrophilic dermatosis (Sweet's syndrome) after influenza vaccination. J Am Acad Dermatol. (2005) 52:367-9. doi: 10.1016/j.jaad.2004.07.061

311. Carpentier O, Piette F, Delaporte E. Sweet's syndrome after BCG vaccination. Acta Derm Venereol. (2002) 82:221. doi: 10.1080/00015550260132604

312. Maddox PR, Motley RJ. Sweet's syndrome: a severe complication of pneumococcal vaccination following emergency splenectomy. Br J Surg. (1990) 77:809-10. doi: 10.1002/bjs.1800770729

313. Radeff B, Harms M. Acute febrile neutrophilic dermatosis (Sweet's syndrome) following BCG vaccination. Acta Derm Venereol. (1986)66:357-8.

314. Wendling D, Leaustic M, Toussirot E, Prati C. Ankylosing spondylitis and Sweet's syndrome. Clin Rheumatol. (2008) 27 (Suppl. 1):S27-8. doi: 10.1007/s10067-007-0829-1

315. Amichai B, Lazarov A, Cagnano M, Halevy S. Sweet's syndrome and chlamydial infection. Aust J Dermatol. (1993) 34:31-3. doi: 10.1111/j.1440-0960.1993.tb00845.x
316. Rubegni P, Marano MR, De Aloe G, Pianigiani E, Bilenchi R, Fimiani M. Sweet's syndrome and Chlamydia pneumoniae infection. J Am Acad Dermatol. (2001) 44:862-4. doi: 10.1067/mjd.2001.112580

317. Tefany FJ, Georgouras K. A neutrophilic reaction of Sweet's syndrome type associated with the oral contraceptive. Aust J Dermatol. (1991) 32:55-9. doi: 10.1111/j.1440-0960.1991.tb00684.x

318. Saez M, Garcia-Bustinduy M, Noda A, Guimera F, Dorta S, Escoda M, et al. Sweet's syndrome induced by oral contraceptive. Dermatology. (2002) 204:84. doi: $10.1159 / 000051820$

319. Kazlouskaya V, Junkins-Hopkins JM, Wu KN, Itenberg SJ. Sweet syndrome caused by oral contraceptives. Int J Dermatol. (2015) 54:e189-91. doi: 10.1111/ijd.12759

320. Huang ZH, Chen HC. Sweet's Syndrome in a Patient with Rheumatoid Arthritis. N Engl J Med. (2017) 377:769. doi: 10.1056/NEJMicm1700945

321. Wang T, Liu Y, Zheng H. Histiocytoid Sweet's syndrome associated with rheumatoid arthritis and pleuritis. Chin Med J. (2014) 127:1396.

322. Gay-Crosier F, Dayer JM, Chavaz P, Hauser C. Rheumatoid neutrophilic dermatitis/sweet's syndrome in a patient with seronegative rheumatoid arthritis. Dermatology. (2000) 201:185-7. doi: 10.1159/000018452

323. Yamauchi PS, Turner L, Lowe NJ, Gindi V, Jackson JM. Treatment of recurrent Sweet's syndrome with coexisting rheumatoid arthritis with the tumor necrosis factor antagonist etanercept. J Am Acad Dermatol. (2006) 54(3 Suppl 2):S122-6. doi: 10.1016/j.jaad.2005.11.1089

324. Coskun U, Gunel N, Senol E, Ilter N, Dursun A, Tuzun D. A case of Sweet's syndrome developed after the treatment of herpes simplex infection in a metastatic breast cancer patient. J Cutaneous Pathol. (2002) 29:301-4. doi: $10.1034 / j .1600-0560.2002 .290508 . x$

325. Oyama Y, Midorikawa S, Satoh K, Sugawara T, Imai H. [Adult onset human herpesvirus (HHV)-7 infection presented necrotizing lymphadenitis and Sweet's syndrome]. Nippon Naika Gakkai Zasshi. (2001) 90:2082-4. doi: 10.2169/naika.90.2082

326. Philips CA, Paramaguru R, Augustine P. Strange case of dimorphic skin rash in a patient with cirrhosis: atypical herpes simplex and sweet's syndrome. BMJ Case Rep. (2017) 20:20. doi: 10.1136/bcr-2017-220743

327. Kitamura H, Kaneko T, Nakano H, Terui K, Ito E, Sawamura D. Juvenile myelomonocytic leukemia presenting multiple painful erythematous lesions diagnosed as Sweet's syndrome. J Dermatol . (2008) 35:368-70. doi: 10.1111/j.1346-8138.2008.00486.x

328. Hoverson AR, Davis MD, Weenig RH, Wolanskyj AP. Neutrophilic dermatosis (Sweet syndrome) of the hands associated with lenalidomide. Arch Dermatol. (2006) 142:1070-1. doi: 10.1001/archderm.142.8.1070-b

329. Thieu KP, Rosenbach M, Xu X, Kist JM. Neutrophilic dermatosis complicating lenalidomide therapy. J Am Acad Dermatol. (2009) 61:709-10. doi: 10.1016/j.jaad.2008.12.011

330. Goette DK. Sweet's syndrome in subacute cutaneous lupus erythematosus. Arch Dermatol. (1985) 121:789-91. doi: 10.1001/archderm.1985.01660060103031

331. Levenstein MM, Fisher BK, Fisher LL, Pruzanski W. Simultaneous occurrence of subacute cutaneous lupus erythematosus and Sweet syndrome. A marker of Sjogren syndrome? Int J Dermatol. (1991) 30:640-3. doi: 10.1111/j.1365-4362.1991.tb03490.x

332. Katayama I. Subacute lupus erythematosus and Sweet syndrome. Int J Dermatol. (1992) 31:165. doi: 10.1111/j.1365-4362.1992. tb03920.x

333. Nayak HK, Vangipuram DR, Kumar S, Kar P, Gupta A, Kapoor N, et al. Sweet's syndrome in a patient with infective endocarditis: a rare clinical entity. BMJ Case Rep. (2012) 20:20. doi: 10.1136/bcr.12.2011.5398

334. Gould KP, Jones JD, Callen JP. Sweet's syndrome in a patient with enterococcal subacute bacterial endocarditis. J Am Acad Dermatol. (2004) 50:798-9. doi: 10.1016/j.jaad.2003.05.003

335. Arias IM, Henning TD, Alba LM, Rubio S. A meningococcal endocarditis in a patient with Sweet's syndrome. Int J Cardiol. (2007) 117:e51-2. doi: $10.1016 /$ j.ijcard.2006.11.088

336. Krilov LR, Jacobson M, Shende A. Acute febrile neutrophilic dermatosis (Sweet's syndrome) presenting as facial cellulitis in a child with juvenile chronic myelogenous leukemia. Pediatr Infect Dis J. (1987) 6:77-9. doi: 10.1097/00006454-198701000-00025 
337. Pintova S, Sidhu H, Friedlander PA, Holcombe RF. Sweet's syndrome in a patient with metastatic melanoma after ipilimumab therapy. Mel Res. (2013) 23:498-501. doi: 10.1097/CMR.0000000000000017

338. Kyllo RL, Parker MK, Rosman I, Musiek AC. Ipilimumab-associated Sweet syndrome in a patient with high-risk melanoma. J Am Acad Dermatol. (2014) 70:e85-e6. doi: 10.1016/j.jaad.2013.11.022

339. Gormley R, Wanat K, Elenitsas R, Giles J, McGettigan S, Schuchter L, et al. Ipilimumab-associated Sweet syndrome in a melanoma patient. J Am Acad Dermatol. (2014) 71:e211-3. doi: 10.1016/j.jaad.2014.06.042

340. Adler NR, Murray WK, Brady B, McCormack C, Pan Y. Sweet syndrome associated with ipilimumab in a patient with metastatic melanoma. Clin Exp Dermatol. (2018) 43:497-9. doi: 10.1111/ced.13399

341. Kalmus Y, Kovatz S, Shilo L, Ganem G, Shenkman L. Sweet's syndrome and subacute thyroiditis. Postgr Med J. (2000) 76:229-30. doi: $10.1136 /$ pmj.76.894.229

342. Dinh H, Murugasu A, Gin D. Sweet's syndrome associated with cellulitis. Aust J Dermatol. (2007) 48:105-9. doi: 10.1111/j.1440-0960. 2007.00347.x

343. Oshitari K, Hida T, Takahashi M, Okada AA, Shiobara T. [Sweet syndrome presenting as orbital cellulitis]. Nippon Ganka Gakkai Zasshi. (2004)108:162-5.

344. Xiao T, He CD, Gao XH, Chen HD. Sweet's syndrome associated with skin methicillin-resistant Staphylococcus epidermidis infection. J Dermatol. (2007) 34:258-61. doi: 10.1111/j.1346-8138.2007.00264.x

345. Soon CW, Kirsch IR, Connolly AJ, Kwong BY, Kim J. Eosinophilrich acute febrile neutrophilic dermatosis in a patient with enteropathyassociated T-cell Lymphoma, Type 1. Am J Dermatopathol. (2016) 38:704-8. doi: 10.1097/DAD.0000000000000549

346. Ayirookuzhi SJ, Ma L, Ramshesh P, Mills G. Imatinib-induced sweet syndrome in a patient with chronic myeloid leukemia. Arch Dermatol. (2005) 141:368-70. doi: 10.1001/archderm.141.3.368

347. Neoh CY, Tan AW, Ng SK. Sweet's syndrome: a spectrum of unusual clinical presentations and associations. Br J Dermatol. (2007) 156:480-5. doi: 10.1111/j.1365-2133.2006.07677.x

348. Nakayama H, Shimao S, Hamamoto T, Munemura C, Nakai A. Neutrophilic dermatosis of the face associated with aortitis syndrome and Hashimoto's thyroiditis. Acta Derm Venereol. (1993) 73:380-1.

349. Medeiros S, Santos R, Carneiro V, Estrela F. Sweet syndrome associated with Hashimoto thyroiditis. Dermat Online J. (2008) 14:10.

350. Francisco CR, Patal PC, Cubillan EA, Isip-Tan IT. Sweet's syndrome associated with Hashimoto's thyroiditis. BMJ Case Rep. (2011) 24:24. doi: 10.1136/bcr.02.2011.3921

351. Bang B, Zachariae C. Capnocytophaga canimorsus sepsis causing Sweet's syndrome. Acta Derm Venereol. (2001) 81:73-4. doi: 10.1080/000155501750208362

352. Gille J, Spieth K, Kaufmann R. Sweet's syndrome as initial presentation of diffuse large B-cell lymphoma. J Am Acad Dermatol. (2002) 46(2 Suppl Case Reports):S11-3. doi: 10.1067/mjd.2002.104970

353. Yorio JT, Mays SR, Ciurea AM, Cohen PR, Wang WL, Hwu WJ, et al. Case of vemurafenib-induced Sweet's syndrome. J Dermatol. (2014) 41:817-20. doi: $10.1111 / 1346-8138.12430$

354. Pattanaprichakul P, Tetzlaff MT, Lapolla WJ, Torres-Cabala CA, Duvic $\mathrm{M}$, Prieto VG, et al. Sweet syndrome following vemurafenib therapy for recurrent cholangiocarcinoma. J Cutan Pathol. (2014) 41:326-8. doi: $10.1111 /$ cup. 12273

355. Cinats AK, Haber RM. Case Report of Sweet's Syndrome associated with autoimmune hepatitis. J Cutaneous Med Surg. (2017) 21:72-4. doi: 10.1177/1203475416665602

356. Yong AS, Lee KY, Murphy J, Phillips M, Rushbrook S, Garioch JJ. Acute febrile neutrophilic dermatosis (Sweet's syndrome) in a patient with biliary sepsis. Postgr Med J. (2013) 89:731-2. doi: 10.1136/postgradmedj-2013-132162

357. Schwartz RA, French SW, Rubenstein DJ, Lambert WC. Acute neutrophilic dermatosis with diffuse histiocytic lymphoma. J Am Acad Dermatol. (1984) 10(2 Pt 2):350-4. doi: 10.1016/S0190-9622(84)80005-5

358. Govindarajan G, Bashir Q, Kuppuswamy S, Brooks C. Sweet syndrome associated with furosemide. Southern Med J. (2005) 98:570-2. doi: 10.1097/01.SMJ.0000157530.63614.9B
359. Chien SM, Jambrosic J, Mintz S. Pulmonary manifestations in Sweet's syndrome: first report of a case with bronchiolitis obliterans organizing pneumonia. Am J Med. (1991) 91:553-4. doi: 10.1016/0002-9343(91)90195-4

360. Longo MI, Pico M, Bueno C, Lazaro P, Serrano J, Lecona M, et al. Sweet's syndrome and bronchiolitis obliterans organizing pneumonia. Am J Med. (2001) 111:80-1. doi: 10.1016/S0002-9343(01)00789-6

361. Martinez W, del Pozo J, Pena C, Yebra-Pimentel MT, Almagro M, Rodriguez-Lozano J, et al. Sweet's syndrome in a woman with chronic dermatophytic infection. Int J Dermatol. (2006) 45:1365-8. doi: 10.1111/j.1365-4632.2006.02890.x

362. Aubin F, Dufour MP, Angonin R, Misery L, Laurent R, Humbert P. Sweet's syndrome associated with cutaneous T cell lymphoma. Eur J Dermatol. (1998) 8:178-9.

363. Keidel S, McColl A, Edmonds S. Sweet's syndrome after adalimumab therapy for refractory relapsing polychondritis. BMJ Case Rep. (2011) 21:21. doi: 10.1136/bcr.10.2011.4935

364. Reid PT, Alderdice J, Carson J, Sinnamon DG. Cryptogenic organizing pneumonia in association with Sweet's syndrome. Resp Med. (1996) 90:57-9. doi: 10.1016/S0954-6111(96)90246-2

365. Tzelepis E, Kampolis CF, Vlachadami I, Moschovi M, Alamani M, Kaltsas G. Cryptogenic organizing pneumonia in Sweet's syndrome: case report and review of the literature. Clin Resp J. (2016) 10:250-4. doi: 10.1111/crj.12206

366. Ruiz AI, Gonzalez A, Miranda A, Torrero V, Gutierrez C, Garcia M. Sweet's syndrome associated with francisella tularensis infection. Int J Dermatol. (2001) 40:791-3. doi: 10.1046/j.1365-4362.2001.01162.x

367. Kuner N, Hartschuh W, Jappe U. Unusual manifestation of Sweet's syndrome in B-cell lymphoma. Acta Derm Venereol. (2003) 83:308-9. doi: 10.1080/00015550310016670

368. Kim YJ, Lee HY, Lee JY, Yoon TY. Interferon beta-1b-induced Sweet's syndrome in a patient with multiple sclerosis. Int J Dermatol. (2015) 54:456-8. doi: 10.1111/j.1365-4632.2012.05840.x

369. Rodriguez-Lojo R, Castineiras I, Juarez Y, Lueiro M, Armesto A, FernandezDiaz ML. Sweet syndrome associated with interferon. Dermat Online J. (2014) 21:13030/qt2006n4sp

370. Kumpfel T, Gerdes LA, Flaig M, Hohlfeld R, Wollenberg A. Drug-induced Sweet's syndrome after mitoxantrone therapy in a patient with multiple sclerosis. Multiple Sclerosis. (2011) 17:495-7. doi: $10.1177 / 1352458510390069$

371. Polat M, Parlak AH, Ors I, Sirmatel F. Erythema nodosum and Sweet's syndrome in patients with glandular tularemia. [Erratum appears in Int J Dermatol. 2011 Nov;50:1444 Note: Mualla, Polat (corrected to Polat, Mualla); Haydar, Parlak Ali (corrected to Parlak, Ali Haydar); Ismail, Ors (corrected to Ors, Ismail); Fatma, Sirmatel (corrected to Sirmatel, Fatma)]. Int J Dermatol. (2011) 50:866-9. doi: 10.1111/j.1365-4632.2010.04647.x

372. Gyorfy A, Kovacs T, Szegedi I, Olah E, Kiss C. Sweet syndrome associated with 13-cis-retinoic acid (isotretinoin) therapy. Med Pediatr Oncol. (2003) 40:135-6. doi: 10.1002/mpo.10089

373. Moghimi J, Pahlevan D, Azizzadeh M, Hamidi H, Pourazizi M. Isotretinoin-associated Sweet's syndrome: a case report. Daru. (2014) 22:69. doi: 10.1186/s40199-014-0069-2

374. Osawa H, Yamabe H, Seino S, Fukushi K, Miyata M, Inuma H, et al. A case of Sjogren's syndrome associated with Sweet's syndrome. Clin Rheumatol. (1997) 16:101-5. doi: 10.1007/BF02238773

375. Kurkcuoglu N, Aksoy F. Sweet's syndrome associated with Helicobacter pylori infection. J Am Acad Dermatol. (1997) 37:123-4. doi: 10.1016/S0190-9622(97)70225-1

376. Nielsen H. Acute febrile neutrophilic dermatosis (Sweet's syndrome) in metastatic breast cancer. Eur J Cancer. (1992) 28A(8-9):1590-1. doi: 10.1016/0959-8049(92)90564-I

377. Antony F, Holden CA. Sweet's syndrome in association with generalized granuloma annulare in a patient with previous breast carcinoma. Clin Exp Dermatol. (2001) 26:668-70. doi: 10.1046/j.1365-2230.2001.00914.x

378. Cohen PR. Proton pump inhibitor-induced Sweet's syndrome: report of acute febrile neutrophilic dermatosis in a woman with recurrent breast cancer. Dermatol Pract Concept. (2015) 5:113-9. doi: 10.5826/dpc.0502a23

379. Teng JM, Draper BK, Boyd AS. Sweet's panniculitis associated with metastatic breast cancer. J Am Acad Dermatol. (2007) 56(2 Suppl):S61-2. doi: 10.1016/j.jaad.2006.05.023 
380. Meulders Q, Allal A, Eggers S, Ferrant A. Sweet's syndrome and myelodysplastic syndrome in a patient with metastatic breast carcinoma. Am J Med. (1989) 86:138-9. doi: 10.1016/0002-9343 (89)90252-0

381. Yamamoto T. Simultaneous occurrence of Sweet's syndrome and erythema nodosum possibly associated with sulfasalazine. Int J Dermatol. (2014) 53:e263-5. doi: 10.1111/ijd.12260

382. Romdhane HB, Mokni S, Fathallah N, Slim R, Ghariani N, Sriha B, et al. Sulfasalazine-induced Sweet's syndrome. Therapie. (2016) 71:345-7. doi: $10.1016 /$ j.therap.2015.11.006

383. Krauser RE, Schumacher HR. The arthritis of Sweet's syndrome. Arth Rheum. (1975) 18:35-41. doi: 10.1002/art.1780180107

384. Takahashi K, Matsui N, Tanabe Y, Yamashita T, Yamaguchi R, Suzuki H. Sweet's syndrome accompanied by joint involvement-a report on two cases. Ryumachi. (1980) 20:4-10.

385. Nolla JM, Juanola X, Valverde J, Roig-Escofet D, Pagerols X, Servitge O. Arthritis in acute febrile neutrophilic dermatosis (Sweet's syndrome). Ann Rheum Dis. (1990) 49:135. doi: 10.1136/ard.49.2.135

386. Halasz CL, Niedt GW, Kurtz CP, Scorpio DG, Bakken JS, Dumler JS. A case of Sweet syndrome associated with human granulocytic anaplasmosis. Arch Dermatol. (2005) 141:887-9. doi: 10.1001/archderm.141.7.887

387. Glendenning J, Khoo V. Sweet's syndrome in prostate cancer. Prostate Cancer Prostatic Dis. (2008) 11:397-8. doi: 10.1038/sj.pcan.4501029

388. Aguiar-Bujanda D, Aguiar-Morales J, Bohn-Sarmiento U. Sweet's syndrome associated with norfloxacin in a prostate cancer patient. QJM. (2004) 97:556. doi: 10.1093/qjmed/hch011

389. Hussein K, Nanda A, Al-Sabah H, Alsaleh QA. Sweet's syndrome (acute febrile neutrophilic dermatosis) associated with adenocarcinoma of prostate and transitional cell carcinoma of urinary bladder. J Eur Acad Dermatol Venereol. (2005) 19:597-9. doi: 10.1111/j.1468-3083.2005.01207.x

390. Dyall-Smith D, Billson V. Sweet's syndrome associated with adenocarcinoma of the prostate. Aust J Dermatol. (1988) 29:25-7. doi: 10.1111/j.1440-0960.1988.tb01221.x

391. Kim J, Choi YJ, Oh SH, Lee KH. A Case of Sweet's panniculitis associated with spinal metastasis from prostate cancer. Ann Dermatol. (2010) 22:478-81. doi: 10.5021/ad.2010.22.4.478

392. Clark BM, Homeyer DC, Glass KR, D'Avignon LC. Clindamycininduced Sweet's syndrome. Pharmacotherapy. (2007) 27:1343-6. doi: 10.1592/phco.27.9.1343

393. Kandula S, Burke WS, Goldfarb JN. Clindamycin-induced Sweet syndrome. J Am Acad Dermatol. (2010) 62:898-900. doi: 10.1016/j.jaad. 2009.03.050

394. Dunn TR, Saperstein HW, Biederman A, Kaplan RP. Sweet syndrome in a neonate with aseptic meningitis. Pediatr Dermatol. (1992) 9:288-92. doi: 10.1111/j.1525-1470.1992.tb00350.x

395. Vaccaro M, Guarneri F, Guarneri C, Borgia F, Cannavo SP. Sweet's syndrome and erythema nodosum after Klebsiella pneumoniae cystitis. Acta Derm Venereol. (2003) 83:290-1. doi: 10.1080/00015550310016562

396. Gimenez-Arnau A, Carles J, Pla-Ferrer C, Barranco CM, Fontane J, Camarasa JG. Sweet's syndrome and malignancy: a case associated with multiple oral squamous-cell carcinoma. Dermatology. (1996) 193:154-5. doi: $10.1159 / 000246236$

397. Tavadia SM, Smith G, Herd RM, Zuk RJ. Sweet's syndrome associated with oral squamous cell carcinoma and exhibiting the Koebner phenomenon. Br J Dermatol. (1998) 141:169-70. doi: 10.1046/j.1365-2133.1999. 02913.x

398. Wallett A, Newland K, Foster-Smith E. Radiation therapy-induced neuroSweet disease in a patient with oral squamous cell carcinoma. Aust J Dermatol. (2017) 58:e51-e3. doi: 10.1111/ajd.12442

399. van der Meij EH, Epstein JB, Hay J, Ho V, Lerner K. Sweet's syndrome in a patient with oral cancer associated with radiotherapy. Eur J Cancer Part B Oral Oncol. (1996) 32B:133-6. doi: 10.1016/0964-1955(95)00070-4

400. Schonfeldt-Lecuona C, Connemann BJ. Sweet's syndrome and polyserositis with clozapine. Am J Psychiatry. (2002) 159:1947. doi: 10.1176/appi.ajp.159.11.1947

401. Kleinen JM, Bouckaert F, Peuskens J. [Clozapine-induced agranulocytosis and Sweet's syndrome in a 74-year-old female patient. A case study]. Tijdschrift Voor Psychiatrie. (2008) 50:119-23.
402. Boivin S, Segard M, Piette F, Delaporte E. Sweet syndrome associated with Pasteurella multocida bronchitis. Arch Int Med. (2000) 160:1869. doi: 10.1001/archinte.160.12.1869

403. Culp L, Crowder S, Hatch S. A rare association of Sweet's syndrome with cervical cancer. Gynecol Oncol. (2004) 95:396-9. doi: 10.1016/j.ygyno.2004.06.050

404. Clark AK, Sarcon AK, Fung MA, Konia T, Laurin EG, Sivamani RK. Malignancy-associated Sweet syndrome: acute febrile neutrophilic dermatosis associated with recurrence of metastatic cervical cancer. Dermat Online J. (2017) 23:15

405. Rondina A, Watson AC. Bullous Sweet's syndrome and pseudolymphoma precipitated by IL-2 therapy. Cutis. (2010) 85:206-13.

406. Eubank KV, Nash J, Duvic M. Sweet syndrome associated with celiac disease. Am J Clin Dermatol. (2009) 10:343-5. doi: 10.2165/11311200-000000000-00000

407. Inomata $\mathrm{N}$, Sasaki $\mathrm{T}$, Nakajima $\mathrm{H}$. Sweet's syndrome with gastric cancer. J Am Acad Dermatol. (1999) 41:1033-4. doi: 10.1016/S0190-9622 (99)70270-7

408. Ikeda Y, Niimi M, Takami H, Kodaira S. Successfully treated carcinoma erysipeloides from gastric cancer. Ann Oncol. (2003) 14:1328-9. doi: 10.1093/annonc/mdg332

409. Uchida H, Ikari Y, Hashizume S, Tokuhashi I, Tadokoro M. A case of Sweet's syndrome with early gastric cancer. Dermatologica. (1990) 181:224-7. doi: 10.1159/000247929

410. Cunquero-Tomas AJ, Ortiz-Salvador JM, Iranzo V, Camps C. Sweet syndrome as the leading symptom in the diagnosis of gastric cancer. Chinese Clin Oncol. (2018) 7:11. doi: 10.21037/cco.2018.01.07

411. Del Giudice P, Vandenbos F, Perrin C, Bernard E, Marq L, Dellamonica P. Sweet's syndrome following abacavir therapy. J Am Acad Dermatol. (2004) 51:474-5. doi: 10.1016/j.jaad.2003.09.033

412. DiCaudo DJ, Ortiz KJ, Mengden SJ, Lim KK. Sweet syndrome (acute febrile neutrophilic dermatosis) associated with pulmonary coccidioidomycosis. Arch Dermatol. (2005) 141:881-4. doi: 10.1001/archderm.141.7.881

413. Nielsen I, Donati D, Strumia R, Zavarini G, Sartori S. Sweet's syndrome and malignancy: report of the first case associated with adenocarcinoma of the lung. Lung Cancer. (1993) 10(1-2):95-9. doi: 10.1016/0169-5002 (93)90314-N

414. Arai H, Rino Y, Yamanaka S, Suganuma N, Yukawa N, Wada N, et al. Lung cancer associated with Sweet's syndrome: report of a case. Surg Today. (2008) 38:639-43. doi: 10.1007/s00595-007-3683-5

415. Yamamoto T, Furuse $Y$, Nishioka K. Sweet's syndrome with small cell carcinoma of the lung. J Dermatol. (1994) 21:125-7. doi: 10.1111/j.1346-8138.1994.tb01428.x

416. Bradley LM, Higgins SP, Thomas MM, Rodney IJ, Halder RM. Sweet syndrome induced by oral acetaminophen-codeine following repair of a facial fracture. Cutis. (2017) 100:E20-3.

417. Yoo WH, Moon SK, Park TS, Baek HS. A case of Sweet's syndrome in patient with dermatomyositis. Korean J Int Med. (1999) 14:78-81. doi: 10.3904/kjim.1999.14.2.78

418. Zillikens D, Goldstein RK, Elsner P, Hartmann AA, Burg G. Sweet's syndrome associated with Salmonella typhimurium infection. Acta Derm Venereol. (1991) 71:77-9.

419. Polimeni G, Cardillo R, Garaffo E, Giardina C, Macri R, Sirna V, et al. Allopurinol-induced Sweet's syndrome. Int J Immunopathol Pharmacol. (2016) 29:329-32. doi: 10.1177/0394632015599705

420. Rodriguez de la Serna A, Domingo-Pedrol P, Blanch-Torra L, PerezPerez A, Obrador-Mayol D. Acute febrile neutrophilic dermatosis (Sweet's syndrome) associated with post-myocardial infarction syndrome (Dressler's syndrome). Arch Int Med. (1985) 145:1522-4. doi: 10.1001/archinte.1985.00360080204032

421. Freitas DF, Valle AC, Cuzzi T, Brandao LG, Zancope-Oliveira RM, Galhardo MC. Sweet syndrome associated with sporotrichosis. Br J Dermatol. (2012) 166:212-3. doi: 10.1111/j.1365-2133.2011.10496.x

422. Fader AN, Winder AD, Sandadi S, Debernardo R. Sweet's syndrome: a cutaneous harbinger of ovarian carcinoma. J Gynecol Oncol. (2012) 23:288-90. doi: 10.3802/jgo.2012.23.4.288

423. Nguyen KQ, Hurst CG, Pierson DL, Rodman OG. Sweet's syndrome and ovarian carcinoma. Cutis. (1983) 32:152-4. 
424. Morgado-Carrasco D, Moreno-Rivera N, Fusta-Novell X, Garcia-Herrera A, Carrera C, Puig S. Histiocytoid Sweet's syndrome during combined therapy with BRAF and MEK inhibitors for metastatic melanoma. Melanoma Res. (2018) 28:256-7. doi: 10.1097/CMR.0000000000000438

425. Oskay T, Anadolu R. Sweet's syndrome in familial Mediterranean fever: possible continuum of the neutrophilic reaction as a new cutaneous feature of FMF. J Cutan Pathol. (2009) 36:901-5. doi: 10.1111/j.1600-0560.2008.01158.x

426. Hara I, Miura T, Yamanaka K, Tanaka K, Yamada Y, Fujisawa M. A case of Sweet's syndrome following septic pulmonary emboli after high-dose chemotherapy for advanced testicular cancer. Int J Urol. (2006) 13:481-4. doi: 10.1111/j.1442-2042.2006.01332.x

427. Shapiro L, Baraf CS, Richheimer LL. Sweet's syndrome (acute febrile neutrophilic dermatosis). Report of a case. Arch Dermatol. (1971) 103:81-4. doi: 10.1001/archderm.1971.04000130083013

428. Sitjas D, Puig L, Cuatrecasas M, De Moragas JM. Acute febrile neutrophilic dermatosis (Sweet's syndrome). Int J Dermatol. (1993) 32:261-8. doi: 10.1111/j.1365-4362.1993.tb04265.x

429. Chebbi W, Berriche O. [Sweet syndrome during pregnancy: a rare entity not to ignore]. Pan Afr Med J. (2014) 18:185.

430. Cohen PR. Pregnancy-associated Sweet's syndrome: world literature review. Obst Gynecol Survey. (1993) 48:584-7. doi: 10.1097/00006254-199308000-00027

431. Giovanna Brunasso AM, Massone C. Clinical images. Sweet syndrome during pregnancy. Can Med Assoc J. (2008) 179:967. doi: $10.1503 / \mathrm{cmaj} .080250$

432. Inoue C, Mochizuki A, Okuda M, Mizoguchi M. [Sweet's syndrome with pregnancy]. Nippon Hifuka Gakkai Zasshi. (1991) 101:973-7.

433. Lopez-Sanchez M, Garcia-Sanchez Y, Marin AP. An unusual evolution of a pregnancy-associated Sweet's Syndrome. Eur J Obst Gynecol Reprod Biol. (2008) 140:283-5. doi: 10.1016/j.ejogrb.2008.01.009

434. Matoses MS, Alcala E, Laguarda M. [Subarachnoid anesthesia for the cesarean section of a patient with Sweet's syndrome related to pregnancy]. Rev Espanola Anestesiol Reanimacion. (2004) 51:111-2.

435. Satra K, Zalka A, Cohen PR, Grossman ME. Sweet's syndrome and pregnancy. J Am Acad Dermatol. (1994) 30(2 Pt 2):297-300. doi: 10.1016/S0190-9622(08)81068-7

436. Pagliarello C, Pepe CA, Lombardi M, Feliciani C, Fabrizi G, Di Nuzzo S. Early miscarriage during Sweet's syndrome: uncommon, but probably not coincidental. Eur J Dermatol. (2013) 23:707-8. doi: 10.1684/ejd.2013.2129

437. Serrano-Falcon C, Serrano-Falcon MM. [Sweet syndrome in a pregnant woman]. Actas Dermsifil. (2010) 101:558-9. doi: 10.1016/S1578-2190(10)70846-5

438. Sankar M, Kaliaperumal K. Pregnancy-Associated Sweet's Syndrome: a rare clinical entity. J Obst Gynaecol India. (2016) 66(Suppl. 2):587-9. doi: 10.1007/s13224-015-0816-4

439. Syed S, Furman J, Nalesnik JG, Basler J. Sweet's syndrome associated with squamous cell carcinoma of the bladder. J Urol. (2003) 170:180-1. doi: 10.1097/01.ju.0000071660.22843.a5

440. Park JY, Park JS, Kim YC. Histiocytoid Sweet's syndrome potentially related to decitabine in a patient with myelodysplastic syndrome. Eur J Dermatol. (2012) 22:811-2.

441. Nakamura S, Nakayama K, Imai T, Ihara C, Ootani A. Sweet's syndrome in a patient with Basedow's disease. J Dermatol. (1988) 15:451-3. doi: 10.1111/j.1346-8138.1988.tb04086.x

442. Phua YS, Al-Ani SA, She RB, de Chalain TM. Sweet's syndrome triggered by scalding: a case study and review of the literature. Burns. (2010) 36:e49-52. doi: 10.1016/j.burns.2009.05.010

443. Rosmaninho A, Machado S, Amorim I, Lobo I, Selores M. Henna tattoo and Sweet's syndrome: a possible relation. Eur J Dermatol. (2009) 19:642-3. doi: 10.1684/ejd.2009.0778

444. Minocha R, Sebaratnam DF, Choi JY. Sweet's syndrome following surgery: cutaneous trauma as a possible aetiological co-factor in neutrophilic dermatoses. Aust J Dermatol. (2015) 56:e74-6. doi: 10.1111/ajd.12142

445. Qiao J, Wang Y, Bai J, Wu Y, Fang H. Concurrence of Sweet's syndrome, pathergy phenomenon and erythema nodosum-like lesions. Anais Brasil Dermatol. (2015) 90:237-9. doi: 10.1590/abd1806-4841.20153201
446. Propst DA, Bossons CR, Sutterlin CE, 3rd. Sweet's syndrome associated with spinal surgical intervention. A case report. Spine. (1998) 23:1708-10.

447. Sherif RD, Harmaty MA, Torina PJ. Sweet Syndrome after bilateral deep inferior epigastric perforator flap breast reconstruction: a case report. Ann Plastic Surg. (2017) 79:e30-e2. doi: 10.1097/SAP.0000000000001157

448. Tortorici S, Buzzanca ML, Burruano F, Difalco P. Sweet's syndrome induced by removable partial denture using a CoCr alloy: case report. Miner Stomatol. (2010) 59:285-7, 8-9.

449. Lipof JS, Beck LA, Reddy SC, Southgate RD, Carney-Young K, Hammert WC. Necrotizing sweet syndrome of the upper extremity after elective hand surgery. J Hand Surg. (2018) 43:389.e1-.e6. doi: 10.1016/j.jhsa.2017.08.019

450. Osband AJ, Laskow DA, Mann RA, Berkowicz T, Ianosi-Irimie M, Boruchoff S. Sweet syndrome after kidney transplantation. Transpl Proc. (2009) 41:1954-6. doi: 10.1016/j.transproceed.2009.03.067

451. Jariwala S, Benson A, Patel P, Friedman A, Broadway K. An Unusual Association between Sweet's Syndrome and Metastatic Papillary Follicular Thyroid Carcinoma. Ann Dermatol. (2013) 25:84-7. doi: 10.5021/ad.2013.25.1.84

452. Guimera FJ, Garcia-Bustinduy M, Noda A, Saez M, Dorta S, Sanchez R, et al. Diazepam-associated Sweet's syndrome. Int J Dermatol. (2010) 39:795-8. doi: 10.1046/j.1365-4362.2000.00051.x

453. O’Brien TJ, Darling JA. Sweet's syndrome and hypothyroidism. Aust J Dermatol. (1994) 35:91-2. doi: 10.1111/j.1440-0960.1994.tb00906.x

454. Tang HKC, Lee DYH, Thompson I, Ingram JR, Stone N, Patel GK. Delayed and localized Sweets syndrome after breast cancer radiotherapy. Breast J. (2018) 24:212-3. doi: 10.1111/tbj.12877

455. Dawe SA, Phillips R, Porter W, Francis NA, Bunker CB. Sweet's syndrome as a complication of radiotherapy for squamous carcinoma of the pharynx. $\mathrm{BrJ}$ Dermatol. (2003) 149:884. doi: 10.1046/j.1365-2133.2003.05541.x

456. Vergara G, Vargas-Machuca I, Pastor MA, Farina MC, Martin L, Requena L. Localization of Sweet's syndrome in radiation-induced locus minoris resistentae. J Am Acad Dermatol. (2003) 49:907-9. doi: 10.1016/S0190-9622(03)01832-2

457. Lee GY, Do MO, Kim SH, Choi HY, Myung KB, Choi YW. Localized Sweet's Syndrome in an Irradiated Field. Ann Dermatol. (2009) 21:300-3. doi: 10.5021/ad.2009.21.3.300

458. Wanat KA, Kovarik CL, Quon H, Miller CJ. Sweet syndrome in a field of radiation therapy. J Am Acad Dermatol. (2012) 67:e284-6. doi: 10.1016/j.jaad.2012.06.042

459. Che Y, Tanioka M, Matsumura Y, Miyachi Y. Splenic irradiationinduced Sweet's syndrome associated with preceding myelofibrosis. Clin Exp Dermatol. (2008) 33:200-1. doi: 10.1111/j.1365-2230.2007. 02589.x

460. Sudhakar MK, Kallarakkal JT, Damodharan J, Sahib K, Mahajan A, Kannan R. Sweet's syndrome preceding carcinoma of the adrenal cortex. I Indian Med Assoc. (2008) 103:433-5.

461. Adler NR, Lin MJ, Cameron R, Gin D. Fluconazole-induced Sweet's syndrome: a novel association. Aust J Dermatol. (2018) 59:e160-e1. doi: 10.1111/ajd.12709

462. Cano A, Ribes R, de la Riva A, Rubio FL, Sanchez C, Sancho JL. Idiopathic hypertrophic cranial pachymeningitis associated with Sweet's Syndrome. Eur J Radiol. (2002) 44:139-42. doi: 10.1016/S0720-048X(02)00090-6

463. Bessis D, Dereure O, Peyron JL, Augias D, Guilhou JJ. Photoinduced Sweet syndrome. Arch Dermatol. (2001) 137:1106-8.

464. Pai VV, Gupta G, Athanikar S, Naveen KN, Sori T, Shastry D. Photoinduced classic sweet syndrome presenting as hemorrhagic bullae. Cutis. (2014) 93:E22-4.

465. Verma R, Vasudevan B, Pragasam V, Mitra D. Unusual Presentation of Idiopathic Sweet's Syndrome in a Photodistributed Pattern. Indian J Dermatol. (2014) 59:186-9. doi: 10.4103/0019-5154.127682

466. Liao A, Danish H, Stoff B, Khan MK. First case of Merkel cell carcinoma in a young patient with Sweet syndrome. Adv Rad Oncol. (2016) 1:122-6. doi: $10.1016 /$ j.adro.2016.03.006

467. Rojas-Perez-Ezquerra P, Noguerado-Mellado B, Saenz de Santamaria Garcia M, Roa-Medellin D, Hernandez-Aragues I, Zubeldia Ortuno JM. Sweet syndrome caused by sensitization to gabapentin. J Allergy Clin Immunol Pract. (2018) 6:685-6. doi: 10.1016/j.jaip.2017.08.019 
468. Altomare G, Capella GL, Frigerio E. Sweet's syndrome in a patient with idiopathic myelofibrosis and thymoma-myasthenia gravisimmunodeficiency complex: efficacy of treatment with etretinate. Haematologica. (1996) 81:54-8.

469. Ainechi S, Carlson JA. Neutrophilic dermatosis limited to lipolymphedematous skin in a morbidly obese woman on dasatinib therapy. Am J Dermatopathol. (2016) 38:e22-6. doi: 10.1097/DAD.00000000000 00358

470. Chu CH, Cheng YP, Kao HL, Liang CW, Chan JY, Yu Y. Lymphedemaassociated neutrophilic dermatosis: Two cases of localized Sweet syndrome on the lymphedematous lower limbs. J Dermatol. (2016) 43:1062-6. doi: $10.1111 / 1346-8138.13379$

471. Demitsu T, Tadaki T. Atypical neutrophilic dermatosis on the upper extremity affected by postmastectomy lymphedema: report of 2 cases. Dermatologica. (1991) 183:230-3. doi: 10.1159/000247677

472. Garcia-Rio I, Perez-Gala S, Aragues M, Fernandez-Herrera J, Fraga J, Garcia-Diez A. Sweet's syndrome on the area of postmastectomy lymphoedema. J Eur Acade Dermatol Venereol. (2006) 20:401-5. doi: 10.1111/j.1468-3083.2006.01460.x

473. Gutierrez-Paredes E, Gonzalez-Rodriguez A, Molina-Gallardo I, JordaCuevas E. Neutrophilic dermatosis on postmastectomy lymphedema. Actas Derm Sifiliogr. (2012) 103:649-51. doi: 10.1016/j.adengl.2012.08.018

474. Lee CH, Lee HC, Lu CF, Hsiao CH, Jee SH, Tjiu JW. Neutrophilic dermatosis on postmastectomy lymphoedema: a localized and less severe variant of Sweet syndrome. Eur J Dermatol. (2009) 19:641-2. doi: 10.1684/ejd.2009.0777

475. Sandlund JT, Miser JS, Miser AW. Sweet syndrome in a patient with osteosarcoma. Am J Clin Oncol. (1996) 19:349-50. doi: 10.1097/00000421-199608000-00005

476. Matzkies FG, Manger B, Schmitt-Haendle M, Nagel T, Kraetsch HG, Kalden JR, et al. Severe septicaemia in a patient with polychondritis and Sweet's syndrome after initiation of treatment with infliximab. Ann Rheum Dis. (2003) 62:81-2. doi: 10.1136/ard.62.1.81

477. Gosheger G, Hillmann A, Ozaki T, Buerger H, Winkelmann W. Sweet's syndrome associated with pigmented villonodular synovitis. Acta Orthopaed Belgica. (2002) 68:68-71.

478. McDermott MB, Corbally MT, O’Marcaigh AS. Extracutaneous Sweet syndrome involving the gastrointestinal tract in a patient with Fanconi anemia. J Pediatr Hematol Oncol. (2001) 23:59-62. doi: 10.1097/00043426-200101000-00015

479. Chatham-Stephens K, Devere T, Guzman-Cottrill J, Kurre P. Metachronous manifestations of Sweet's syndrome in a neutropenic patient with Fanconi anemia. Pediatr Blood Cancer. (2008) 51:128-30. doi: 10.1002/pbc. 21538

480. Giulino L, Guinan EC, Gillio AP, Drachtman RA, Teruya-Feldstein J, Boulad F. Sweet syndrome in patients with Fanconi anaemia: association with extracutaneous manifestations and progression of haematological disease. Br J Haematol. (2011) 154:278-81. doi: 10.1111/j.1365-2141. 2011.08604.x

481. Baron F, Sybert VP, Andrews RG. Cutaneous and extracutaneous neutrophilic infiltrates (Sweet syndrome) in three patients with Fanconi anemia. $J$ Pediatr. (1989) $115(5 \mathrm{Pt} 1): 726-9$. doi: 10.1016/S0022-3476(89)80649-3

482. Dereure O, Ebrard-Charra S, Guillon F, Baldet P, Guilhou JJ. Sweet's syndrome associated with pheochromocytoma. Dermatology. (2004) 208:175. doi: 10.1159/000076499

483. Baquerizo Nole KL, Lee E, Villada G, Romanelli P. Ketoconazole-induced Sweet syndrome: a new association. Am J Dermatopathol. (2015) 37:419-22. doi: 10.1097/DAD.0000000000000252

484. del Pozo J, Martinez W, Carro E, Arevalo MP, Rodriguez-Lozano J, Fonseca E. A case of Sweet's syndrome and pemphigus vulgaris. J Eur Acad Dermatol Venereol. (2004) 18:745-6. doi: 10.1111/j.1468-3083.2004.01056.x

485. Wong GA, Guerin DM, Parslew R. Sweet's syndrome and polycythaemia rubra vera. Clin Exp Dermatol. (2000) 25:296-8. doi: 10.1046/j.1365-2230.2000.00646.x

486. Gomez Vazquez M, Sanchez-Aguilar D, Peteiro C, Toribio J. Sweet's syndrome and polycythaemia vera. J Eur Acad Dermatol Venereol. (2005) 19:382-3. doi: 10.1111/j.1468-3083.2005.01091.x
487. Moreira AP, Souza FF, Gaspar NK, Quattrino AL, Vilar EA. [Sweet's syndrome associated with polycythemia vera]. Anais Brasileiros Dermatol. (2009) 84:663-6. doi: 10.1590/S0365-05962009000600014

488. Furukawa T, Takahashi M, Shimada H, Moriyama Y, Shibata A, Katsumi S. Polycythaemia vera with Sweet's syndrome. Clin Laboratory Haematol. (1989) 11:67-70. doi: 10.1111/j.1365-2257.1989.tb00177.x

489. Cox NH, Leggat H. Sweet's syndrome associated with polycythemia rubra vera. J Am Acad Dermatol. (1990) 23(6 Pt 1):1171-2. doi: 10.1016/S0190-9622(08)80922-X

490. O’Connor Reina C, Garcia Iriarte MT, Rodriguez Diaz A, Gomez Angel D, Garcia Monge E, Sanchez Conejo-Mir J. Tonsil cancer and Sweet's syndrome. Otolaryngology. (2008) 119:709-10. doi: 10.1016/S0194-5998(98) 70042-7

491. Shabtaie SA, Tan NY, Parikh RS, Papadakis KA. Concurrent Sweet's syndrome and myopericarditis following mesalamine therapy. BMJ Case Rep. (2018) 2018:1-4. doi: 10.1136/bcr-2017-223851

492. Elinav H, Maly A, Ilan Y, Rubinow A, Naparstek Y, Amital H. The coexistence of Sweet's syndrome and Still's disease-is it merely a coincidence? J Am Acad Dermatol. (2004) 50(5 Suppl):S90-2. doi: 10.1016/S0190-9622(03)02796-8

493. Su WP, Alegre VA, White WL. Myelofibrosis discovered after diagnosis of Sweet's syndrome. Int J Dermatol. (1990) 29:201-4. doi: 10.1111/j.1365-4362.1990.tb03800.x

494. Chatterjee B, Rqieh U, Greaves P, Piras D, Firth J, Saja K. Sweet syndrome as terminal event in ruxolitinib-treated myelofibrosis. Br J Haematol. (2015) 169:307. doi: 10.1111/bjh.13334

495. Delgado P, Franco E, Sanchez M. [Sweet's syndrome and pyoderma gangrenosum in a patient with idiopathic myelofibrosis]. Sangre. (1993) 38:248-9.

496. Liu Y, Tabarroki A, Billings S, Visconte V, Rogers HJ, Hasrouni E, et al. Successful use of very low dose subcutaneous decitabine to treat high-risk myelofibrosis with Sweet syndrome that was refractory to 5-azacitidine. Leukemia Lymphoma. (2014) 55:447-9. doi: 10.3109/10428194.2013.802315

497. Sakoda T, Kanamitsu Y, Mori Y, Sasaki K, Yonemitsu E, Nagae K, et al. Recurrent Subcutaneous Sweet's Disease in a myelofibrosis patient treated with ruxolitinib before allogeneic stem cell transplantation. Int Med. (2017) 56:2481-5. doi: 10.2169/internalmedicine.8491-16

498. Geelkerken RH, Lagaay MB, van Deijk WA, Spaander PJ. Sweet syndrome associated with liposarcoma: a case report. Netherlands J Med. (1994)45:107-9.

499. Hamill M, Bowling J, Vega-Lopez F. Sweet's syndrome and a Mirena intrauterine system. J Family Plan Reprod Health Care. (2004) 30:115-6. doi: 10.1783/147118904322995519

500. Itoh H, Shimasaki S, Nakashima A, Ohsato K, Tokikuni N, Kitajima C. Sweet's syndrome associated with subacute necrotizing lymphadenitis. Int Med. (1992) 31:686-9. doi: 10.2169/internalmedicine.31.686

501. Sedel D, Huguet P, Lebbe C, Donadieu J, Odievre M, Labrune P. Sweet syndrome as the presenting manifestation of chronic granulomatous disease in an infant. Pediatr Dermatol. (1994) 11:237-40. doi: 10.1111/j.1525-1470.1994.tb00593.x

502. Knipstein JA, Ambruso DR. Sweet syndrome in an infant with chronic granulomatous disease. J Pediatr Hematol Oncol. (2012) 34:372-4. doi: 10.1097/MPH.0b013e3182346be1

503. O’Regan GM, Ho WL, Limaye S, Keogan MT, Murphy GM. Sweet's syndrome in association with common variable immunodeficiency. Clin Exp Dermatol. (2009) 34:192-4. doi: 10.1111/j.1365-2230.2008.02814.x

504. Jindal R, Jain A, Mittal A, Shirazi N. Sweet's syndrome as the presenting manifestation of gall bladder adenocarcinoma. BMJ Case Rep. (2012) 12:12. doi: 10.1136/bcr-2012-006869

505. Bachmeyer C, Begon E, Blum L, Cerf I, Petitjean B, VignonPennamen $\mathrm{MD}$, et al. Overlapping neutrophilic dermatosis in a patient with SAPHO syndrome. Arch Dermatol. (2007) 143:275-6. doi: 10.1001/archderm.143.2.275

506. Beretta-Piccoli BC, Sauvain MJ, Gal I, Schibler A, Saurenmann T, Kressebuch $\mathrm{H}$, et al. Synovitis, acne, pustulosis, hyperostosis, osteitis (SAPHO) syndrome in childhood: a report of ten cases and review of the literature. Eur J Pediatr. (2000) 159:594-601. doi: 10.1007/s004310000500

507. Sobol UA, Sherman KL, Smith J, Nagda SN, Micetich K, Nickoloff BJ, et al. Sweet's syndrome with neurologic manifestations in a patient with 
esophageal adenocarcinoma: case report and review of the literature. Int $J$ Dermatol. (2009) 48:1062-5. doi: 10.1111/j.1365-4632.2009.04138.x

508. Retief CR, Malkinson FD. Nitrofurantoin-associated Sweet's syndrome. Cutis. (1999) 63:177-9.

509. Rattan R, Sharma A, Shanker V, Tegta GR, Verma GK. Transient autoimmune thyroiditis associated with Sweet's syndrome. Indian J Dermatol Venereol Leprol. (2016) 82:112. doi: 10.4103/0378-6323.162323

510. Mali-Gerrits MG, Rampen FH. Acute febrile neutrophilic dermatosis (Sweet's syndrome) and adenocarcinoma of the rectum. Clin Exp Dermatol. (1988) 13:105-6. doi: 10.1111/j.1365-2230.1988.tb00669.x

511. Morita Y, Ogura T, Yamamura M, Makino H, Ota Z, Morishita Y. Sweet's syndrome associated with undifferentiated connective tissue syndrome. Ann Rheum Dis. (1995) 54:937-8. doi: 10.1136/ard.54.11.937

512. Miller RM, Darben TA, Nedwich J, Savige J. Propylthiouracil-induced antineutrophil cytoplasmic antibodies in a patient with Graves' disease and a neutrophilic dermatosis. Br J Dermatol. (1999) 141:943-4. doi: 10.1046/j.1365-2133.1999.03186.x

513. Choi HS, Kim HJ, Lee TH, Lee SH, Lee TW, Ihm CG, et al. Quinupristin/dalfopristin-induced Sweet's syndrome. Korean J Int Med. (2003) 18:187-90. doi: 10.3904/kjim.2003.18.3.187

514. Ikram S, Veerappan Kandasamy V. Ticagrelor-induced Sweet Syndrome: an unusual dermatologic complication after percutaneous coronary intervention. Cardiovasc Intervent Therap. (2017) 32:244-6. doi: 10.1007/s12928-016-0398-9

515. Dickson EL, Bakhru A, Chan MP. Topotecan-induced Sweet's syndrome: A case report. Gynecol Oncol Case Rep. (2013) 4:50-2. doi: 10.1016/j.gynor.2013.01.002

516. Martinez Andres B, Sastre Lozano V, Sanchez Melgarejo JF. Sweet syndrome after treatment with vedolizumab in a patient with Crohn's disease. Revista Espanola de ENFERMEDADEs Digestivas. (2018) 110:530. doi: 10.17235/reed.2018.5603/2018

517. Cohen PR. Sweet's syndrome-a comprehensive review of an acute febrile neutrophilic dermatosis. Orph J Rare Dis. (2007) 2:34.

518. Parsapour K, Reep MD, Gohar K, Shah V, Church A, Shwayder TA. Familial Sweet's syndrome in 2 brothers, both seen in the first 2 weeks of life. J Am Acad Dermatol. (2003) 49:132-8. doi: 10.1067/mjd.2003.328

519. von den Driesch P. Sweet's syndrome (acute febrile neutrophilic dermatosis). J Am Acad Dermatol. (1994) 31:535-56; quiz 57-60. doi: 10.1016/S0190-9622(94)70215-2

520. Costello MJ, Canizares O, Montague M, Buncke CM. Cutaneous manifestations of myelogenous leukemia. Arch Dermatol. (1955) 71:605-14. doi: 10.1001/archderm.1955.01540290045009

521. Cohen PR, Kurzrock R. Sweet's syndrome and cancer. Clin Dermatol. (1993) 11:149-57. doi: 10.1016/0738-081X(93)90112-P

522. Ghoufi L, Ortonne N, Ingen-Housz-Oro S, Barhoumi W, Begon E, Haioun C, et al. Histiocytoid sweet syndrome is more frequently associated with myelodysplastic syndromes than the classical neutrophilic variant: a comparative series of 62 patients. Medicine. (2016) 95:e3033. doi: 10.1097/MD.0000000000003033

523. Nelson CA, Noe MH, McMahon CM, Gowda A, Wu B, Ashchyan HJ, et al. Sweet syndrome in patients with and without malignancy: a retrospective analysis of 83 patients from a tertiary academic referral center. J Am Acad Dermatol. (2018) 78:303-9.e4. doi: 10.1016/j.jaad.2017.09.013

524. Kawakami T, Ohashi S, Kawa Y, Takahama H, Ito M, Soma Y, et al. Elevated serum granulocyte colony-stimulating factor levels in patients with active phase of sweet syndrome and patients with active behçet disease: implication in neutrophil apoptosis dysfunction. Arch Dermatol. (2004) 140:570-4. doi: 10.1001/archderm.140.5.570

525. Fiehn C, Wermann M, Pezzutto A, Hufner M, Heilig B. [Plasma GM-CSF concentrations in rheumatoid arthritis, systemic lupus erythematosus and spondyloarthropathy]. Z Rheumatol. (1992) 51:121-6.

526. Nausch N, Lundtoft C, Schulz G, Henckel H, Mayatepek E, Fleischer B, et al. Multiple cytokines for the detection of Mycobacterium tuberculosis infection in children with tuberculosis. Int J Tubercul Lung Dis. (2017) 21:270-7. doi: 10.5588/ijtld.16.0351

527. Suzuki A, Takahashi T, Okuno Y, Seko S, Fukuda Y, Nakamura K, et al. Liver damage in patients with colony-stimulating factor-producing tumors. Am J Med. (1993) 94:125-32. doi: 10.1016/0002-9343(93)90173-M
528. Kowalska M, Tajer J, Chechlinska M, Fuksiewicz M, Kotowicz B, Kaminska J, et al. Serum macrophage colony-stimulating factor (M-CSF) in patients with Hodgkin lymphoma. Med Oncol. (2012) 29:2143-7. doi: 10.1007/s12032-011-0010-1

529. Omori F, Okamura S, Shimoda K, Otsuka T, Harada M, Niho Y. Levels of human serum granulocyte colony-stimulating factor and granulocytemacrophage colony-stimulating factor under pathological conditions. Biotherapy. (1992) 4:147-53. doi: 10.1007/BF02171759

530. Dereure O, Hillaire-Buys D, Guilhou JJ. Neutrophil-dependent cutaneous side-effects of leucocyte colony-stimulating factors: manifestations of a neutrophil recovery syndrome? Brit J Dermatol. (2004) 150:1228-30. doi: 10.1111/j.1365-2133.2004.06007.x

531. Reuss-Borst MA, Muller CA, Waller HD. The possible role of G-CSF in the pathogenesis of Sweet's syndrome. Leukemia Lymphoma. (1994) 15:261-4. doi: 10.3109/10428199409049722

532. Wallach D, Vignon-Pennamen MD. From acute febrile neutrophilic dermatosis to neutrophilic disease: forty years of clinical research. J Am Acad Dermatol. (2006) 55:1066-71. doi: 10.1016/j.jaad.2006.07.016

533. Shinojima Y, Toma Y, Terui T. Sweet syndrome associated with intrahepatic cholangiocarcinoma producing granulocyte colony-stimulating factor. Brit $J$ Dermatol. (2006) 155:1103-4. doi: 10.1111/j.1365-2133.2006.07521.x

534. van Kamp H, van den Berg E, Timens W, Kraaijenbrink RA, Halie MR, Daenen SM. Sweet's syndrome in myeloid malignancy: a report of two cases. Brit J Haematol. (1994) 86:415-7. doi: 10.1111/j.1365-2141. 1994.tb04757.x

535. Magro CM, Kiani B, Li J, Crowson AN. Clonality in the setting of Sweet's syndrome and pyoderma gangrenosum is not limited to underlying myeloproliferative disease. J Cutan Pathol. (2007) 34:526-34. doi: 10.1111/j.1600-0560.2006.00654.x

536. Buck T, Gonzalez LM, Lambert WC, Schwartz RA. Sweet's syndrome with hematologic disorders: a review and reappraisal. Int J Dermatol. (2008) 47:775-82. doi: 10.1111/j.1365-4632.2008.03859.x

537. Van Loon K, Gill RM, McMahon P, Chigurupati R, Siddiqi I, Fox L, et al. 20q- clonality in a case of oral sweet syndrome and myelodysplasia. Am J Clin Pathol. (2012) 137:310-5. doi: 10.1309/AJCP9I7NRWYLTJHV

538. Sujobert P, Cuccuini W, Vignon-Pennamen D, Martin-Garcia N, Albertini AF, Uzunov M, et al. Evidence of differentiation in myeloid malignancies associated neutrophilic dermatosis: a fluorescent in situ hybridization study of 14 patients. J Investig Dermatol. (2013) 133:1111-4. doi: 10.1038/jid.2012.408

539. Mo W, Wang X, Wang Y, Li Y, Zhang R. Clonal neutrophil infiltrates in concurrent Sweet's syndrome and acute myeloid leukemia: a case report and literature review. Cancer Genet. (2018) 226-227:11-6. doi: 10.1016/j.cancergen.2018.04.120

540. Fathi AT, Le L, Hasserjian RP, Sadrzadeh H, Levis M, Chen YB. FLT3 inhibitor-induced neutrophilic dermatosis. Blood. (2013) 122:239-42. doi: 10.1182/blood-2013-01-478172

541. Varadarajan N, Boni A, Elder DE, Bagg A, Micheletti R, Perl AE, et al. FLT3 inhibitor-associated neutrophilic dermatoses. JAMA Dermatol. (2016) 152:480-2. doi: 10.1001/jamadermatol.2015.6121

542. Pemmaraju N, Kantarjian H, Ravandi F, Cortes J. FLT3 inhibitors in the treatment of acute myeloid leukemia: the start of an era? Cancer. (2011) 117:3293-304. doi: 10.1002/cncr.25908

543. von den Driesch P. Sweet's syndrome and vasculitis. J Am Acad Dermatol. (1996) 34:539. doi: 10.1016/S0190-9622(96)90470-3

544. Villarreal-Villarreal CD, Ocampo-Candiani J, Villarreal-Martinez A. Sweet syndrome: a review and update. Actas Dermosifiliogr. (2016) 107:369-78. doi: 10.1016/j.ad.2015.12.001

545. Horio T. Photoaggravation of acute febrile neutrophilic Dermatosis (sweet's syndrome). J Dermatol. (1985) 12:191-4. doi: 10.1111/j.1346-8138.1985.tb01558.x

546. Meyer V, Schneider SW, Bonsmann G, Beissert S. Experimentally confirmed induction of Sweet's syndrome by phototesting. Acta Dermatovenereol. (2011) 91:720-1. doi: 10.2340/00015555-1139

547. Johannemann D, Brunner M, Zouboulis CC. Facial Sweet syndrome mimicking photoallergic contact dermatitis. Journal der Deutschen Dermatologischen Gesellschaft. (2009) 7:697-700. doi: 10.1111/j.1610-0387.2009.07044.x 
548. Natkunarajah J, Gordon K, Chow J, Sarkany R, Millington GW, Marsden RA. Photoaggravated Sweet's syndrome. Clin Exp Dermatol. (2010) 35:e18-9. doi: 10.1111/j.1365-2230.2009.03329.x

549. Belhadjali H, Marguery MC, Lamant L, Giordano-Labadie F, Bazex J. Photosensitivity in Sweet's syndrome: two cases that were photoinduced and photoaggravated. Brit J Dermatol. (2003) 149:675-7. doi: 10.1046/j.1365-2133.2003.05487.x

550. Yoshizumi M, Nakamura T, Kato M, Ishioka T, Kozawa K, Wakamatsu $\mathrm{K}$, et al. Release of cytokines/chemokines and cell death in UVBirradiated human keratinocytes, HaCaT. Cell Biol Int. (2008) 32:1405-11. doi: 10.1016/j.cellbi.2008.08.011

551. Strickland I, Rhodes LE, Flanagan BF, Friedmann PS. TNF-alpha and IL-8 are upregulated in the epidermis of normal human skin after UVB exposure: correlation with neutrophil accumulation and E-selectin expression. $J$ Investig Dermatol. (1997) 108:763-8. doi: 10.1111/1523-1747.ep12292156

552. Lyck R, Enzmann G. The physiological roles of ICAM-1 and ICAM2 in neutrophil migration into tissues. Curr Opin Hematol. (2015) 22:53-9. doi: 10.1097/MOH.0000000000000103

553. Stucki A, Rivier AS, Gikic M, Monai N, Schapira M, Spertini O. Endothelial cell activation by myeloblasts: molecular mechanisms of leukostasis and leukemic cell dissemination. Blood. (2001) 97:2121-9. doi: 10.1182/blood.V97.7.2121

554. Vignon-Pennamen MD, Aractingi S. Sweet's syndrome and leukemia cutis: a common skin homing mechanism? Dermatology. (2003) 206:81-4. doi: $10.1159 / 000068462$

555. Dominguez ER, Greene JN, Sandin RL, DeGregorio R, Glass LF. Sweet syndrome and leukemia cutis in the same patient: a case report and review. Cancer Control J Moffitt Cancer Center. (1995) 2:343-6. doi: 10.1177/107327489500200413

556. del Pozo J, Martinez W, Pazos JM, Yebra-Pimentel MT, Garcia Silva J, Fonseca E. Concurrent Sweet's syndrome and leukemia cutis in patients with myeloid disorders. Int J Dermatol. (2005) 44:677-80. doi: 10.1111/j.1365-4632.2005.02037.x

557. Giasuddin AS, El-Orfi AH, Ziu MM, El-Barnawi NY. Sweet's syndrome: is the pathogenesis mediated by helper $\mathrm{T}$ cell type 1 cytokines? J Am Acad Dermatol. (1998) 39:940-3. doi: 10.1016/S0190-9622(98) 70266-X

558. Antiga E, Maglie R, Volpi W, Bianchi B, Berti E, Marzano AV, et al. T helper type 1-related molecules as well as interleukin-15 are hyperexpressed in the skin lesions of patients with pyoderma gangrenosum. Clin Exp Immunol. (2017) 189:383-91. doi: 10.1111/cei.12989

559. Marzano AV, Fanoni D, Antiga E, Quaglino P, Caproni M, Crosti C, et al. Expression of cytokines, chemokines and other effector molecules in two prototypic autoinflammatory skin diseases, pyoderma gangrenosum and Sweet's syndrome. Clin Exp Immunol. (2014) 178:48-56. doi: 10.1111/cei.12394

560. Marzano AV, Cugno M, Trevisan V, Fanoni D, Venegoni L, Berti E, et al. Role of inflammatory cells, cytokines and matrix metalloproteinases in neutrophil-mediated skin diseases. Clin Exp Immunol. (2010) 162:100-7. doi: 10.1111/j.1365-2249.2010.04201.x

561. Caproni M, Antiga E, Volpi W, Verdelli A, Venegoni L, Quaglino P, et al. The Treg/Th17 cell ratio is reduced in the skin lesions of patients with pyoderma gangrenosum. Brit J Dermatol. (2015) 173:275-8. doi: 10.1111/bjd. 13670

562. Marzano AV, Cugno M, Trevisan V, Lazzari R, Fanoni D, Berti E, et al. Inflammatory cells, cytokines and matrix metalloproteinases in amicrobial pustulosis of the folds and other neutrophilic dermatoses. Int J Immunopathol Pharmacol. (2011) 24:451-60. doi: 10.1177/039463201102400218

563. Fitch E, Harper E, Skorcheva I, Kurtz SE, Blauvelt A. Pathophysiology of psoriasis: recent advances on IL-23 and Th17 cytokines. Curr Rheumatol Rep. (2007) 9:461-7. doi: 10.1007/s11926-007-0075-1

564. Patel DD, Kuchroo VK. Th17 cell pathway in human immunity: lessons from genetics and therapeutic interventions. Immunity. (2015) 43:1040-51. doi: 10.1016/j.immuni.2015.12.003

565. Lauridsen HM, Pellowe AS, Ramanathan A, Liu R, MillerJensen K, McNiff JM, et al. Tumor necrosis factor-alpha and IL-17A activation induces pericyte-mediated basement membrane remodeling in human neutrophilic dermatoses. Am J Pathol. (2017) 187:1893-906. doi: 10.1016/j.ajpath.2017.04.008

566. Yamagata T, Skepner J, Yang J. Targeting Th17 Effector cytokines for the treatment of autoimmune diseases. Arch Immunol Ther Exp. (2015) 63:40514. doi: 10.1007/s00005-015-0362-x

567. Liu R, Lauridsen HM, Amezquita RA, Pierce RW, Jane-Wit D, Fang C, et al. IL-17 promotes neutrophil-mediated immunity by activating microvascular pericytes and not endothelium. J Immunol. (2016) 197:2400-8. doi: 10.4049/jimmunol.1600138

568. Reuss-Borst MA, Pawelec G, Saal JG, Horny HP, Muller CA, Waller HD. Sweet's syndrome associated with myelodysplasia: possible role of cytokines in the pathogenesis of the disease. Brit J Haematol. (1993) 84:356-8. doi: 10.1111/j.1365-2141.1993.tb03083.x

569. Uhara H, Saida T, Nakazawa H, Ito T. Neutrophilic dermatoses with acute myeloid leukemia associated with an increase of serum colony-stimulating factor. J Am Acad Dermatol. (2008) 59(2 Suppl 1):S10-2. doi: 10.1016/j.jaad.2007.08.026

570. Gasteiger G, Rudensky AY. Opinion: interactions of innate and adaptive lymphocytes. Nat Rev Immunol. (2014) 14:631-9. doi: 10.1038/NRI3726

571. Bourke JF, Berth-Jones J, Graham-Brown RA. Sweet's syndrome responding to cyclosporin. Brit J Dermatol. (1992) 127:368. doi: 10.1111/j.1365-2133.1992.tb14823.x

572. von den Driesch P, Steffan C, Zobe A, Hornstein OP. Sweet's syndrome-therapy with cyclosporin. Clin Exp Dermatol. (1994) 19:274-7. doi: 10.1111/j.1365-2230.1994.tb01187.x

573. Yasuda F, Fujio Y, Kakuta R, Funakoshi T, Amagai M, Ohyama M. Use of cyclosporin A for successful management of steroid-resistant Sweet's syndrome patient with possible myelodysplastic syndrome. J Dermatol. (2014) 41:465-6. doi: 10.1111/1346-8138.12469

574. Calixto R, Menezes Y, Ostronoff M, Sucupira A, Botelho LF, Florencio R, et al. Favorable outcome of severe, extensive, granulocyte colonystimulating factor-induced, corticosteroid-resistant Sweet's syndrome treated with high-dose intravenous immunoglobulin. J Clin Oncol. (2014) 32:e1-2. doi: 10.1200/JCO.2011.40.3212

575. Seminario-Vidal L, Guerrero C, Sami N. Refractory Sweet's syndrome successfully treated with rituximab. JAAD Case Rep. (2015) 1:123-5. doi: 10.1016/j.jdcr.2015.03.002

576. Hashemi SM, Fazeli SA, Vahedi A, Golabchifard R. Rituximab for refractory subcutaneous Sweet's syndrome in chronic lymphocytic leukemia: a case report. Mol Clin Oncol. (2016) 4:436-40. doi: 10.3892/mco. 2015.715

577. Takahama H, Kanbe T. Neutrophilic dermatosis of the dorsal hands: a case showing HLA B54, the marker of Sweet's syndrome. Int J Dermatol. (2010) 49:1079-80. doi: 10.1111/j.1365-4632.2009.04422.x

578. Costa-Reis P, Sullivan KE. Chronic recurrent multifocal osteomyelitis. J Clin Immunol. (2013) 33:1043-56. doi: 10.1007/s10875-013-9902-5

579. Ferguson PJ, Chen S, Tayeh MK, Ochoa L, Leal SM, Pelet A, et al. Homozygous mutations in LPIN2 are responsible for the syndrome of chronic recurrent multifocal osteomyelitis and congenital dyserythropoietic anaemia (Majeed syndrome). J Med Genet. (2005) 42:551-7. doi: 10.1136/jmg.2005.030759

580. Miyoshi T, Yamashita K, Ohno T, Izumi T, Takaori-Kondo A, Sasada M, et al. Familial Mediterranean fever gene as a possible modifier of Sweet syndrome with chronic myelogenous leukemia. Acta Haematol. (2008) 120:57-62. doi: 10.1159/000158578

581. Masters SL, Lagou V, Jeru I, Baker PJ, Van Eyck L, Parry DA, et al. Familial autoinflammation with neutrophilic dermatosis reveals a regulatory mechanism of pyrin activation. Sci Transl Med. (2016) 8:332ra45. doi: 10.1126/scitranslmed.aaf1471

582. Heilig R, Broz P. Function and mechanism of the pyrin inflammasome. Eur J Immunol. (2018) 48:230-8. doi: 10.1002/eji.201746947

583. Snyder R, Libby T, Raciti P, Amin B, Jacobson M, Rakheja D, et al. Myelodysplastic syndrome and sweet's syndrome are associated with a mutation in isocitrate dehydrogenase 1. Anticancer Res. (2018) 38:2201-5. doi: 10.21873/anticanres.12462

584. Lu C, Ward PS, Kapoor GS, Rohle D, Turcan S, Abdel-Wahab O, et al. IDH mutation impairs histone demethylation and results in a block to cell differentiation. Nature. (2012) 483:474-8. doi: 10.1038/nature10860 
585. Lorenz U. SHP-1 and SHP-2 in T cells: two phosphatases functioning at many levels. Immunol Rev. (2009) 228:342-59. doi: 10.1111/j.1600-065X.2008.00760.x

586. Oka T, Ouchida M, Koyama M, Ogama Y, Takada S, Nakatani Y, et al. Gene silencing of the tyrosine phosphatase SHP1 gene by aberrant methylation in leukemias/lymphomas. Cancer Res. (2002) 62:6390-4.

587. Beghini A, Ripamonti CB, Peterlongo P, Roversi G, Cairoli R, Morra E, et al. RNA hyperediting and alternative splicing of hematopoietic cell phosphatase (PTPN6) gene in acute myeloid leukemia. Hum Mol Genet. (2000) 9:2297-304. doi: 10.1093/oxfordjournals.hmg.a018921

588. Ma XZ, Jin T, Sakac D, Fahim S, Zhang X, Katsman Y, et al. Abnormal splicing of SHP-1 protein tyrosine phosphatase in human $\mathrm{T}$ cells. Implications for lymphomagenesis. Experimental Hematol. (2003) 31:131-42. doi: 10.1016/S0301-472X(02)01025-1

589. Oka T, Yoshino T, Hayashi K, Ohara N, Nakanishi T, Yamaai Y, et al. Reduction of hematopoietic cell-specific tyrosine phosphatase SHP-1 gene expression in natural killer cell lymphoma and various types of lymphomas/leukemias : combination analysis with cDNA expression array and tissue microarray. Am J Pathol. (2001) 159:1495-505. doi: 10.1016/S0002-9440(10)62535-7

590. Nesterovitch AB, Szanto S, Gonda A, Bardos T, Kis-Toth K, Adarichev $\mathrm{VA}$, et al. Spontaneous insertion of a b2 element in the ptpn6 gene drives a systemic autoinflammatory disease in mice resembling neutrophilic dermatosis in humans. Am J Pathol. (2011) 178:170114. doi: 10.1016/j.ajpath.2010.12.053

591. Nesterovitch AB, Gyorfy Z, Hoffman MD, Moore EC, Elbuluk N, Tryniszewska B, et al. Alteration in the gene encoding protein tyrosine phosphatase nonreceptor type 6 (PTPN6/SHP1) may contribute to neutrophilic dermatoses. Am J Pathol. (2011) 178:1434-41. doi: 10.1016/j.ajpath.2010. 12.035

592. Cohen PR, Kurzrock R. Sweet's syndrome: a review of current treatment options. Am J Clin Dermatol. (2002) 3:11731. doi: 10.2165/00128071-200203020-00005

593. Cohen PR. Sweet's syndrome - a comprehensive review of an acute febrile neutrophilic dermatosis. Orphanet J. Rare Dis. (2007) 2:34. doi: 10.1186/1750-1172-2-34

594. Honma K, Saga K, Onodera H, Takahashi M. Potassium iodide inhibits neutrophil chemotaxis. Acta Dermatovenereol. (1990) 70:247-9.

595. Paschke S, Weidner AF, Paust T, Marti O, Beil M, Ben-Chetrit E. Technical advance: Inhibition of neutrophil chemotaxis by colchicine is modulated through viscoelastic properties of subcellular compartments. J Leukocyte Biol. (2013) 94:1091-6. doi: 10.1189/jlb.1012510
596. Schadt CR, Callen JP. Management of neutrophilic dermatoses. Dermatol Ther. (2012) 25:158-72. doi: 10.1111/j.1529-8019.2012. 01488.x

597. Delluc A, Limal N, Puechal X, Frances C, Piette JC, Cacoub P. Efficacy of anakinra, an IL1 receptor antagonist, in refractory Sweet syndrome. Ann Rheumat Dis. (2008) 67:278-9. doi: 10.1136/ard.2006. 068254

598. Herlin T, Fiirgaard B, Bjerre M, Kerndrup G, Hasle H, Bing X, et al. Efficacy of anti-IL-1 treatment in Majeed syndrome. Ann Rheumat Dis. (2013) 72:410-3. doi: 10.1136/annrheumdis-2012-201818

599. Kluger N, Gil-Bistes D, Guillot B, Bessis D. Efficacy of anti-interleukin1 receptor antagonist anakinra (Kineret ${ }^{\circledR}$ ) in a case of refractory Sweet's syndrome. Dermatology. (Basel, Switzerland). (2011) 222:1237. doi: $10.1159 / 000326112$

600. Agarwal A, Barrow W, Selim MA, Nicholas MW. Refractory subcutaneous sweet syndrome treated with adalimumab. JAMA Dermatol. (2016) 152:8424. doi: 10.1001/jamadermatol.2016.0503

601. Moreno Marquez C, Maldonado Perez B, Castro Laria L. Infliximab as rescue treatment in sweet's syndrome related to corticodependent ulcerative colitis. J Crohns Colitis. (2018) 12:755-6. doi: 10.1093/ecco-jcc/jjy018

602. Foster EN, Nguyen KK, Sheikh RA, Prindiville TP. Crohn's disease associated with Sweet's syndrome and Sjogren's syndrome treated with infliximab. Clin Dev Immunol. (2005) 12:145-9. doi: 10.1080/174025205001 34254

603. Rahier JF, Lion L, Dewit O, Lambert M. Regression of Sweet's syndrome associated with Crohn's disease after anti-Tumour Necrosis Factor therapy. Acta Gastroenterol Belg. (2005) 68:376-9.

604. Fujii A, Mizutani Y, Hattori Y, Takahashi T, Ohnishi H, Yoshida $\mathrm{S}$, et al. Sweet's syndrome successfully treated with granulocyte and monocyte adsorption apheresis. Case Rep Dermatol. (2017) 9:13-8. doi: 10.1159/000475878

Conflict of Interest Statement: The authors declare that the research was conducted in the absence of any commercial or financial relationships that could be construed as a potential conflict of interest.

Copyright (c) 2019 Heath and Ortega-Loayza. This is an open-access article distributed under the terms of the Creative Commons Attribution License (CC BY). The use, distribution or reproduction in other forums is permitted, provided the original author(s) and the copyright owner(s) are credited and that the original publication in this journal is cited, in accordance with accepted academic practice. No use, distribution or reproduction is permitted which does not comply with these terms. 